\title{
Effective properties of an isotropic solid weakened by micro-cracks located at inter-granular boundaries
}

\author{
Joane Meynard ${ }^{\mathrm{a}}$, Marcelle Ibrahim ${ }^{\mathrm{a}}$, Arnaud Monnier ${ }^{\mathrm{a}}$, Michel Bornert ${ }^{\mathrm{c}}$, Etienne \\ Castelier $^{\mathrm{a}}$, Christelle Duguay ${ }^{\mathrm{a}}$, Mihail Garajeu ${ }^{\mathrm{b}}$, Renaud Masson ${ }^{\mathrm{a}, *}$ \\ ${ }^{a}$ CEA, DES, IRESNE, DEC, Cadarache F-13108 Saint-Paul-lez-Durance, France \\ ${ }^{b}$ Aix Marseille Univ, CNRS, Centrale Marseille, LMA, F-13453 Marseille, France \\ ${ }^{c}$ Laboratoire Navier, Ecole des ponts, Univ. Gustave Eiffel, CNRS, 6-8 avenue Blaise Pascal, \\ Champs-sur-Marne, 77455 Marne-la-Vallée Cedex
}

\begin{abstract}
This study presents a new methodology for estimating the effective properties of solids containing cracks along the inter-granular boundaries, using analytical developments and numerical simulations. The latter are based on the generation of virtual microstructures of such type obtained by superimposing a Voronoï tessellation modeling the granular network with a random dispersion of overlapping spheres in 3-D, or disks in 2-D, which serve to locate the cracks at the inter-granular boundaries. The different features of this microstructure model are studied herein, especially the morphological effects induced by varying the size ratio between grains and spheres/disks. By means of full-field simulations, the effective thermal conductivities of the generated microstructures are estimated and compared with those of uniformly weakened solids (presenting uniform crack dispersion). For the latter microstructures, the Ponte-Castañeda \& Willis (1995) upper bound turns out to be close to the full-field results. In addition, the full-field computations show that the spatial distribution of inter-granular cracks induces a dramatic degradation of the effective thermal conductivity. Modifying only the cut-off crack density in the mathematical expression of the Ponte Castañeda and Willis bound provides a relevant analytical estimate of the effective conductivity of solids weakened by inter-granular cracks. This cut-off crack density only depends on the microstructural parameters. This new estimate is shown to improve the one derived by Sevostianov \& Kachanov (2019) and based on the differential scheme at least for the microstructures considered herein. Finally, new estimates of the moduli of elasticity for isotropic cracked solids weakened at inter-granular boundaries are also provided. The effective bulk modulus thus estimated for 3-D solids is shown to remain below the upper bound which can also be generated by injecting the effective conductivity predicted by full-field computations into the classical cross-property relations.
\end{abstract}

Keywords: Cracks, Thermal conductivity, Elasticity, Homogenization, Fast Fourier Transform, Microstructures, Inter-granular boundaries

\footnotetext{
${ }^{*}$ Corresponding author

Email address: renaud.masson@cea.fr (Renaud Masson)
} 


\section{Introduction}

Inter-granular boundaries play an important role in the mechanical and physical properties of solids. Here, the inter-granular boundaries represent weak zones where micro-cracks preferentially appear as the result of the manufacturing process or more generally due to mechanical or even chemical solicitations. Therefore, these cracks may deeply affect the considered solids and their properties because of their specific locations. In this article, we aim at modeling by homogenization the effective properties of such isotropic solid weakened by micro-cracks located at the inter-granular boundaries.

Various homogenization models have been proposed in the past to estimate the effective properties of micro-cracked solids. Some of these models are presented herein for the thermal conduction problem but equivalent results exist of course for other effective properties such as elasticity. By neglecting the effect of crack interactions, the "non-interacting crack" model can be used to simply derive the effective conductivity of a solid as a function of the crack concentration. The case of circular (or ribbon) cracks was firstly investigated by Bristow (1960). When the crack concentration is low, this model coincides with the "dilute scheme" which was extended by Shafiro \& Kachanov (2000) to cover more general elliptical shapes and angular distributions. For non dilute concentrations, Budiansky \& O'Connell (1976) proposed improved estimates based on the self-consistent approach in the case of elliptical cracks randomly oriented and distributed. To deliver Hashin-Shtrikman-like estimates of the effective conductivity taking into account the effects of the shape/orientation and the spatial distribution of cracks in a separate manner, Duan et al. (2006) extended the theory initially proposed by Ponte-Castañeda \& Willis (1995) for elasticity and applied it to different heterogeneous microstructures in thermal conduction problems including the case of cracks randomly distributed in a solid. Starting from the differential scheme Hashin (1988), Sevostianov \& Kachanov (2019) very recently proposed an estimate of the effective conductivity of polycrystalline material weakened by inter-granular cracks.

Full-field computations are nowadays intensively used to compute the effective properties of representative volume elements (RVE) of heterogeneous solids. In Grechka et al. (2006), RVE computations with the finite-element method were used to study the influence of the shape of randomly oriented cracks on the effective thermal conductivity. Pouya \& Ghoreychi (2001) also used this finite-element method to compute the strength of micro-cracked solids. As the Fast-Fourier Transform technique (FFT, Moulinec \& Suquet (1998)) has been proved to be particularly efficient to perform RVE computations, this method has been increasingly used to compute the effective properties of heterogeneous materials including the case of micro-cracked solids ( $\mathrm{Li}$ et al. (2012b), Li et al. (2012a)). However, with this FFT technique, surface objects like cracks have to be modeled by albeit small but non zero volumes so that particular attention must be paid to such computations. Gasnier et al. (2018) recently proposed a general method to assess the bias induced by FFT computations in the case of micro-cracked solids. For three-dimensional elasticity problems, these authors showed that with the backward-and-forward finite-difference Fourier scheme (BF-FFT, Willot \& Pellegrini (2008)), relevant estimates of the effective properties are yielded with a crack thickness larger than 1.5 voxels.

RVE computations have proved to be very interesting for studying the effect of the spatial distribution of phases in a solid (inclusions, voids, ...) on the effective properties. For 
example, considering a voided matrix, Bilger et al. (2005) studied the effect of the spatial distribution of voids on the simulated effective properties by modeling microstructures with spherical-void clustering where either connected or disconnected clusters are deliberately introduced. These clusters were obtained by superimposing two sets of spheres randomly distributed with very different radii and retaining only those small voids whose centers either belong to the larger spheres for the non-connected clusters case, or do not belong to these spheres for the dual connected case. In this manner, the larger spheres played as limits of the clustering areas. This last superimposition technique is well-suited to represent the preferential location of cracks along inter-granular boundaries (IGB) in a given solid.

In line with the superimposition technique, we generated microstructures weakened at their inter-granular boundaries in order to evaluate the impact of the crack location on the effective properties. The effective properties of those microstructures can be compared with those of solids with randomly distributed cracks (hereinafter referred to as "uniformly weakened solids"). For these uniformly weakened solids, some theoretical bounds and estimates proposed in the past to model their effective conductivity and moduli are reviewed in section 2. Section 3 details the principles used for generating the synthetic microstructures of solids weakened uniformly or at their inter-granular boundaries. Two-dimensional (2-D) and three-dimensional (3-D) microstructures have been generated. In section 4 , the effective thermal conductivity of these microstructures is estimated using FFT computations. The effect of the crack location on the effective conductivity is especially studied. Comparisons with analytical predictions including the original and recent work of Sevostianov \& Kachanov (2019) led us to propose a new estimate of the effective conductivity of solids with inter-granular cracks. Morphological effects related to the grain and crack sizes are also investigated in this section. Finally, theoretical results related to the prediction of the effective moduli of elastic solids weakened at inter-granular boundaries are inferred from the results related to the thermal conduction issue, which are reported in section 5 .

\section{Effective properties of cracked media}

Next results are given for 3-D and 2-D micro-cracked microstructures with $V$ and $S$ being the Representative Volume and Surface Element respectively. Hereinafter, the spatial dimension is denoted $d$ with $d=2$ for 2-D cases and $d=3$ for 3-D cases. Data related to the solid phase is written using an upper index ${ }^{(1)}$ while the upper index ${ }^{(2)}$ is related to cracks. The behavior of the solid phase is isotropic with $\sigma^{(1)}$ denoting its conductivity while $\left(\mu^{(1)}, k^{(1)}\right)$ denote its shear and bulk moduli of elasticity, respectively.

This section is organized as follows: the commonly used crack density parameter is defined in section 2.1, then some classical results related to bounds and estimates of the effective conductivity and elastic moduli of cracked solids are given in section 2.2. The estimate proposed by Sevostianov \& Kachanov (2019) in the case of solids weakened at their intergranular boundaries is also reported in section 2.2. Finally, comparisons of these different approaches are reported in section 2.3. 


\subsection{Crack density parameter}

Cracks can be viewed as the limiting case of voided inclusions distributed in a matrix, with vanishing thermal conductivity $\sigma^{(2)}$ or moduli $\left(\mu^{(2)}, k^{(2)}\right)$, when some aspect ratio tends to a limit such that their volume fraction tends to zero. For 3-D microstructures, the pennyshaped cracks considered hereafter are defined as disks with an aspect ratio $\omega^{(2)}=e /\left(2 R_{c}\right)$ approaching 0 where $\left(2 R_{c}\right)$ and $e$ represent the disk diameter and thickness respectively. For 2-D microstructures, cracks (also called ribbon-cracks) are defined as rectangles with an aspect ratio $\omega^{(2)}=e /\left(2 R_{c}\right)$ approaching 0 where $\left(2 R_{c}\right)$ and $e$ represent the length and the width respectively. When $N_{c}$ denotes the number of cracks in $V$ in 3-D (or in $S$ in 2-D), the crack concentration $c^{(2)}$ (i.e. the volume fraction for 3-D cases or the surface fraction in 2-D cases) equals $N_{c} e \pi R_{c}^{2} / V$ in 3-D (or $2 N_{c} e R_{c} / S$ in 2-D) and will tend toward zero as the crack thickness $e$ decreases.

Based on this limit, the conventional bounds on the effective property of a heterogeneous solid yield little information. For instance the effective conductivity $\tilde{\sigma}$ must be larger than the lower bounds provided by Wiener (1912) and Hashin \& Shtrikman (1962) (here equivalent to the Maxwell lower bound Maxwell (1873)) and given by:

$$
\begin{aligned}
\tilde{\sigma} & \geq \sigma^{(2)}\left(\frac{(d-1) \sigma^{(2)}+\sigma^{(1)}+\left(1-c^{(2)}\right)(d-1)\left(\sigma^{(1)}-\sigma^{(2)}\right)}{(d-1) \sigma^{(2)}+\sigma^{(1)}+\left(1-c^{(2)}\right)\left(\sigma^{(2)}-\sigma^{(1)}\right)}\right) \quad \text { (Maxwell-) } \\
& \left.\geq \frac{1}{\left(1-c^{(2)}\right) / \sigma^{(1)}+c^{(2)} / \sigma^{(2)}} \quad \text { (Wiener- }\right)
\end{aligned}
$$

However and as previously remarked by Gibiansky \& Torquato (1996b) for the bulk modulus, these two lower bounds are indeterminate ${ }^{1}$ when both $c^{(2)}$ and $\sigma^{(2)}$ tend toward zero.

Of course, even if the number $N_{c}$ of cracks in $V$ is known, more needs to be known about their shape and spatial distribution. For a $3-\mathrm{D}$ case with $N_{c}$ isolated cracks of diameter $2 R_{c}$ embedded in a volume $V$, their density can be evaluated with the dimensionless crack density $\rho$ introduced by Bristow (1960) and defined as:

$$
\rho=\frac{N_{c} R_{c}^{3}}{V}=\frac{1}{2 \pi} \frac{c^{(2)}}{\omega^{(2)}}
$$

In addition, by defining the crack area per unit of volume $A_{V}^{(2)}$ as:

$$
A_{V}^{(2)}=\frac{\pi N_{c} R_{c}^{2}}{V}
$$

the penny-shaped crack density reads equivalently as:

$$
\rho=\frac{R_{c}}{\pi} A_{V}^{(2)}
$$

\footnotetext{
${ }^{1}$ The Wiener and Hashin-Shtrikman upper bounds coincide in that particular case and yields the trivial result $\tilde{\sigma} \leq \sigma^{(1)}$.
} 
In 2-D cases, the concentration of $N_{c}$ ribbon-cracks of length $\left(2 R_{c}\right)$ in a surface $S$ can also be evaluated with the dimensionless ribbon-crack density $\rho$ used by Sevostianov et al. (2004) and defined as:

$$
\rho=\frac{N_{c} R_{c}{ }^{2}}{S}=\frac{1}{4} \frac{c^{(2)}}{\omega^{(2)}}
$$

Using the definition of the ribbon-crack length per unit of area $\left(L_{A}^{(2)}=2 N_{c} R_{c} / S\right)$, the ribbon-crack density can also be expressed as:

$$
\rho=\frac{R_{c}}{2} L_{A}^{(2)}
$$

At this stage, it is worth remarking that the definitions presented above are valid for isolated cracks. Therefore, as soon as cracks are interconnected, they cannot be considered as isolated and no longer present a uniform length. However, the crack area per unit of volume $A_{V}^{(2)}$ can still be defined and measured. The expression of $A_{V}^{(2)}$ (equation (4)) can then be used to define in a conventional manner the number $N_{c}$ of equivalent penny-shaped isolated cracks of an arbitrarily chosen diameter $l_{c}$. Equation (3) applied with this new definition of $N_{c}$ and substitution of $R_{c}$ with $\left(l_{c} / 2\right)$ therefore leads to an expression of the crack density for solids with interconnected cracks. By making similar modifications to equation (7), an expression of the crack-density for overlapping cracks in 2-D cases can also be obtained, once the length of the equivalent ribbon cracks is chosen.

\subsection{Existing models to predict the effective thermal and elastic properties}

Some useful theoretical models are presented below for an isotropic spatial distribution of isolated and randomly oriented ribbon-cracks $(d=2)$ and penny-shaped cracks $(d=3)$. Apart from the Ponte-Castañeda \& Willis (1995) bounds, different approximations can be found in the literature. In Gibiansky \& Torquato (1996b), the self-consistent (Budiansky \& O'Connell (1976)), the differential (Hashin (1988)) and the non-interacting approximations (Bristow (1960)) are detailed for both the effective conductivity and the effective moduli.

Sevostianov \& Kachanov (2019) were able to derive new estimates based on the differential approximation for cracks no longer uniformly distributed in the solid but located at their inter-granular boundaries. To the best of our knowledge, these are the only estimates proposed so far that predict the effective properties of solids weakened at their inter-granular boundaries. This recent contribution is also reported at the end of this section.

\subsection{1. $P C W$ upper bounds}

As mentioned in the introduction, Ponte-Castañeda \& Willis (1995) extended the HashinShtrikman bound by taking into account the effects of the cracks shape and spatial distribution separately. For non-overlapping penny-shaped cracks randomly oriented and distributed in an isotropic manner, the upper bound for the effective bulk modulus $\tilde{k}^{\mathrm{PCW}+}(\rho)$ reads:

$$
\frac{\tilde{k}^{\mathrm{PCW}+}(\rho)}{k^{(1)}}=\frac{1-\rho / \rho_{k(d)}^{\mathrm{PCW}+}}{1+\left(\beta_{k(d)}-1 / \rho_{k(d)}^{\mathrm{PCW}+}\right) \rho}
$$


where the effective bulk modulus becomes zero when $\rho=\rho_{k(d)}^{\mathrm{PCW}+}$. Following Kachanov (1992), this critical crack density value is called the cut-off crack density. It will depend on the space dimension $(d)$, the model (here the PCW upper bound), the crack shape and the considered property (here the bulk modulus). For $3-\mathrm{D}$ media $(d=3)$ with penny-shaped cracks, the two scalar coefficients $\left(\beta_{k(3)}, \rho_{k(3)}^{\mathrm{PCW}+}\right)$ read:

$$
\beta_{k(3)}=\frac{16}{9} \frac{1-\left(\nu^{(1)}\right)^{2}}{1-2 \nu^{(1)}} \quad \text { and } \quad \rho_{k(3)}^{\mathrm{PCW}+}=\frac{27}{32}\left(\frac{1}{1+\nu^{(1)}}\right)
$$

where $\nu^{(1)}$ is the Poisson ratio of the solid phase and is given by $\left(3 k^{(1)}-2 \mu^{(1)}\right) /\left(6 k^{(1)}+2 \mu^{(1)}\right)$ or equivalently $k^{(1)} / \mu^{(1)}=2\left(1+\nu^{(1)}\right) /\left(3\left(1-2 \nu^{(1)}\right)\right)$. When the crack density is low, the effective bulk modulus can be approximated fairly well by:

$$
\tilde{k}^{\mathrm{PCW}+}(\rho) / k^{(1)} \approx 1-\beta_{k(d)} \rho
$$

which shows that the coefficient $\beta_{k(d)}$ drives the variation of the effective bulk modulus with the crack density near zero. This coefficient depends on the space dimension, the moduli and the crack shape (here penny-shaped cracks).

At this stage, it is worth recalling that the PCW bound is a rigorous bound for a specific class of cracked isotropic media such that the two-point correlation functions characterizing the spatial distribution of the crack centers exhibit an isotropic symmetry. This condition limits the maximal crack density, namely $\rho \leq \delta_{d} / \pi$ with $\delta_{2}=1$ for 2 -D media while $\delta_{3}=3 / 4$ for 3-D media. This maximum value corresponds to the onset of overlapping of the so-called "security spheres" (see Ponte-Castañeda \& Willis (1995)) surrounding cracks and is much lower than the cut-off point $\rho_{k(d)}^{\mathrm{PCW}+}$. Therefore, this upper bound is only an estimate beyond these critical values and more generally can not be considered as a bound for any isotropic cracked media.

This PCW bound was later applied to determine the effective conductivity (see Duan et al. (2006)) and reads:

$$
\frac{\tilde{\sigma}^{\mathrm{PCW}+}(\rho)}{\sigma^{(1)}}=\frac{1-\rho / \rho_{(d)}^{\mathrm{PCW}+}}{1+\left(\beta_{d}-1 / \rho_{(d)}^{\mathrm{PCW}+}\right) \rho}
$$

where $\rho_{(d)}^{\mathrm{PCW}+}$ is the cut-off point predicted by the model in a $d$-D case and that is such as $1 / \rho_{(d)}^{\mathrm{PCW}+}=\beta_{d}(d-1) / d$. The additional constant $\beta_{d}$ equals $\pi / 2$ (for $d=2$ ) and $8 / 9$ (for $d=3)$. It is remarked that the slope at $\rho=0$ for the effective bulk modulus $\left(-\beta_{k(d)}\right)$ is related to the slope $\left(-\beta_{d}\right)$ associated with the effective conductivity:

$$
\beta_{k(d)}=2 \beta_{d}\left(\frac{1-\left(\nu^{(1)}\right)^{2}}{1-2 \nu^{(1)}}\right)
$$

\subsubsection{Self-consistent estimate}

For 2-D and 3-D media, the self-consistent estimate of the effective conductivity was first established by Hoenig (1983) and is given by:

$$
\tilde{\sigma}^{\mathrm{SC}}(\rho) / \sigma^{(1)}=1-\beta_{d} \rho
$$


It is worth remarking that this expression coincides with the PCW upper bound for low crack density values. In addition, the same relation (13) applies to the dilute scheme. This surprising result (also reported for instance in Gibiansky \& Torquato (1996a)) is closely related to the fact that the thermal gradient within the inclusion does not depend on the conductivity of the isotropic infinite medium when considering a fully insulating ellipsoidal inclusion embedded in an infinite volume submitted to a given thermal gradient at infinity.

For 3-D media, the self-consistent estimate of the bulk modulus ${ }^{2}$ is given by:

$$
\frac{\tilde{k}^{\mathrm{SC}}(\rho)}{k^{(1)}}=1-\frac{16}{9} \frac{1-\bar{\nu}^{2}}{1-2 \bar{\nu}} \rho
$$

(see Budiansky \& O'Connell (1976), relations (36)) where $\bar{\nu}$ is solution of the following equation (see Budiansky \& O’Connell (1976), relation $\left(42^{\prime}\right)$ ):

$$
\rho=\frac{45}{16} \frac{\left(\nu^{(1)}-\bar{\nu}\right)(2-\bar{\nu})}{\left(1-\bar{\nu}^{2}\right)\left(10 \nu^{(1)}-\bar{\nu}\left(1+3 \nu^{(1)}\right)\right)}
$$

As $\bar{\nu} \approx \nu^{(1)}$ when the crack density $\rho$ is close to zero, a direct expression of the self-consistent estimate of the bulk modulus for small values of the crack density is:

$$
\tilde{k}^{\mathrm{SC}}(\rho) / k^{(1)} \approx 1-\beta_{k(3)} \rho
$$

which coincides with the first-order expansion of the PCW upper bound. Hereafter, we will no longer use the expressions of the effective bulk and shear moduli for 2-D cases, but these expressions can be easily found in several papers (e.g. Gibiansky \& Torquato (1996b)).

\subsubsection{Non-interacting approximation}

The non-interacting approximation for the effective conductivity was initially proposed by Bristow (1960). For randomly oriented cracks in an isotropic solid, this approximation denoted by $\tilde{\sigma}^{\mathrm{NI}}(\rho)$ reads:

$$
\frac{\tilde{\sigma}^{\mathrm{NI}}(\rho)}{\sigma^{(1)}}=\frac{1}{1+\beta_{d} \rho}
$$

General expressions of the effective moduli as predicted by this non-interaction approximation are reported in Gibiansky \& Torquato (1996b). Moreover, this model coincides with the estimates delivered by the Mori \& Tanaka (1973) model applied to cracked solids. Here below, we only recall the expression of the effective bulk modulus for $2-\mathrm{D}$ or $3-\mathrm{D}$ cases:

$$
\frac{\tilde{k}^{\mathrm{NI}}(\rho)}{k^{(1)}}=\frac{1}{1+\beta_{k(d)} \rho}
$$

Once again, these approximations of the effective conductivity and bulk modulus coincide with the self-consistent estimate and the PCW bound when the crack density tends to zero, as expected with such situations with vanishing interactions between cracks.

\footnotetext{
${ }^{2} \mathrm{~A}$ similar expression of the effective shear modulus is reported in Budiansky \& O'Connell (1976) (see relation $\left(44^{\prime}\right)$ in this reference).
} 


\subsubsection{Differential scheme}

The differential model is derived incrementally by gradual addition of infinitesimal amounts of inclusions (here the cracks), the effect of a crack density increment on the effective property being given by the dilute model described above. For the effective conductivity and a given crack density interval $[u ; u+d u]$, this differential relation is simply:

$$
\tilde{\sigma}^{\mathrm{DS}}(u+d u)=\tilde{\sigma}^{\mathrm{DS}}(u)\left(1-\beta_{d} d u\right)
$$

for wich it is recalled that $\beta_{d}$ do not depend on $\tilde{\sigma}^{\mathrm{DS}}(u)$ (see section 2.2.2) so that by integration on $u$, the final effective conductivity up to a given crack density $\rho$ reads:

$$
\tilde{\sigma}^{\mathrm{DS}}(\rho) / \sigma^{(1)}=e^{-\beta_{d} \rho}
$$

Similar expressions have been derived for the moduli Hashin (1988). For the effective bulk modulus, it reads:

$$
\frac{\tilde{k}^{\mathrm{DS}}(\rho)}{k^{(1)}}=\frac{1-2 \nu^{(1)}}{1-2 \bar{\nu}}\left(\frac{\bar{\nu}}{\nu^{(1)}}\right)^{10 / 9}\left(\frac{3-\nu^{(1)}}{3-\bar{\nu}}\right)^{1 / 9}
$$

where $\bar{\nu}$ depends on the crack density, the Poisson coefficient of the solid and is the solution of the nonlinear equation:

$$
\frac{5}{8} \ln \left(\nu^{(1)} / \bar{\nu}\right)+\frac{15}{64} \ln \left(\frac{1-\bar{\nu}}{1-\nu^{(1)}}\right)+\frac{45}{128} \ln \left(\frac{1+\bar{\nu}}{1+\nu^{(1)}}\right)+\frac{5}{128} \ln \left(\frac{3-\bar{\nu}}{3-\nu^{(1)}}\right)=\rho
$$

\subsubsection{Solids weakened at their inter-granular boundaries}

Recently, Sevostianov \& Kachanov (2019) proposed a model to derive the effective properties of a solid weakened by inter-granular cracks. This model is derived from the differential scheme. It has only been formulated for 3-D cases but the corresponding estimate for the effective conductivity can be easily formulated for 2-D and 3-D cases such as:

$$
\tilde{\sigma}(\rho) / \sigma^{(1)}=\left(1-\rho / \rho_{\text {sat }(d)}^{I G B}\right)^{\beta_{d} \rho_{\text {sat }(d)}^{I G B}}
$$

In this last expression, $\rho_{\text {sat }(d)}^{I G B}$ denotes the crack density for which all the inter-granular boundaries are cracked. As expected, the effective conductivity is zero when the crack density reaches this limit value. We will see in the next sections that this microstructural parameter can be estimated for synthetic microstructures (section 3) and plays a key role in the effective properties (sections 4 and 5). In addition and as reported by the authors, the initial slope equals $\left(-\beta_{d}\right)$ and is consistent not only with the one of the PCW bound but also with the estimates presented above. Although less convenient to implement, similar estimates of the bulk and shear moduli are also proposed by these authors (see Sevostianov \& Kachanov (2019) for more details). Explicit expressions of the Poisson ratio and the Young modulus are also provided in this work. As this model predicts the behavior of solids weakened at their inter-granular boundaries (and the others focus on uniformly weakened solids), we do not consider this model in the comparisons presented below in 2.3, but we do consider the estimates delivered by this model in section 4 . 


\subsection{Effective properties of solids uniformly weakened by cracks: comparisons of the different theoretical predictions}

The PCW bound and the different approximations presented in section 2.2 to model the effective conductivity of solids uniformly weakened by cracks were used in Figure 1 to plot the evolution of the effective conductivity normalized by the conductivity of the solid phase as a function of the crack density. Similar trends are observed for the 2-D (Figure 1(a)) and 3-D cases (Figure 1(b)).

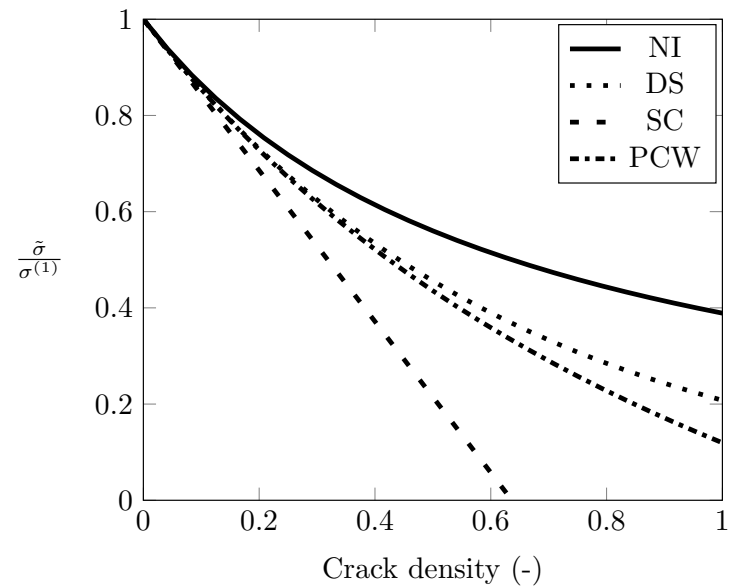

(a)

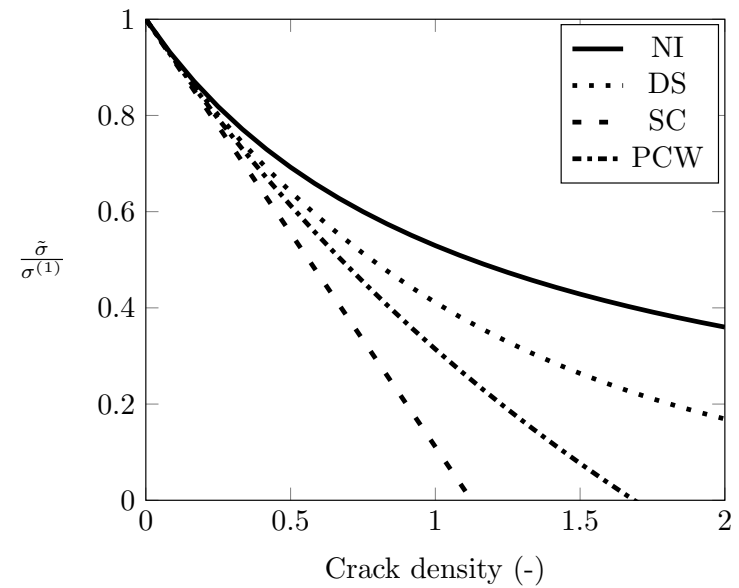

(b)

Figure 1: Normalized effective conductivity as a function of the crack density in 2-D (Figure (a)) and 3-D cases (Figure (b)). Comparison between analytical estimates given by the non-interacting crack model (NI, continuous line), the differential scheme (DS, loosely dotted curve), the self-consistent estimate(sc, loosely dashed curve)identical to the dilute model and the PCW upper bound Ponte-Castañeda \& Willis (1995) (PCw, dot-dashed line).

First, the curve for the self-consistent approximation lies below the PCW curve. For larger crack density values, the self-consistent, the differential and the non-interacting estimates deviate significantly and the curve corresponding to the non-interacting crack approximation lies above that for the PCW bound even for a crack density lower than $\rho=1 / \pi \approx 0.32$ (in 2 -D) or $\rho=3 /(4 \pi) \approx 0.24$ (in 3 -D), for which this model yields a rigorous upper bound at least for the specific class of microstructures defined in 2.2.1. Beyond this value, this upper bound is only an estimate. Furthermore, the non-interacting crack approximation and the differential scheme model do not predict any cut-off and therefore lead to much higher predictions for the effective conductivity than the other two models.

In Figure 2, these estimates and the PCW bound of the effective bulk modulus of 3-D media are plotted as a function of the crack density (for $\nu^{(1)}=0.3$ ). Results as provided by the self-consistent estimate are slightly lower as compared to that for the PCW upper bound. This trend is similar to that already reported in Ponte-Castañeda \& Willis (1995). The results delivered by the two approximations are also very close to those given by the PCW upper bound if the crack density remains below 0.1. However and as remarked for the effective conductivity, the predictions yielded by the non-interacting approximation and 
the differential scheme violate the PCW bound (for $\rho \geq 0.1$ and $\rho \leq 0.24$ ). This deficiency of the non-interacting crack approximation (or equivalently the Mori-Tanaka estimate) and the differential scheme is also reported in Ponte-Castañeda \& Willis (1995) for a non dilute dispersion of randomly oriented cracks.

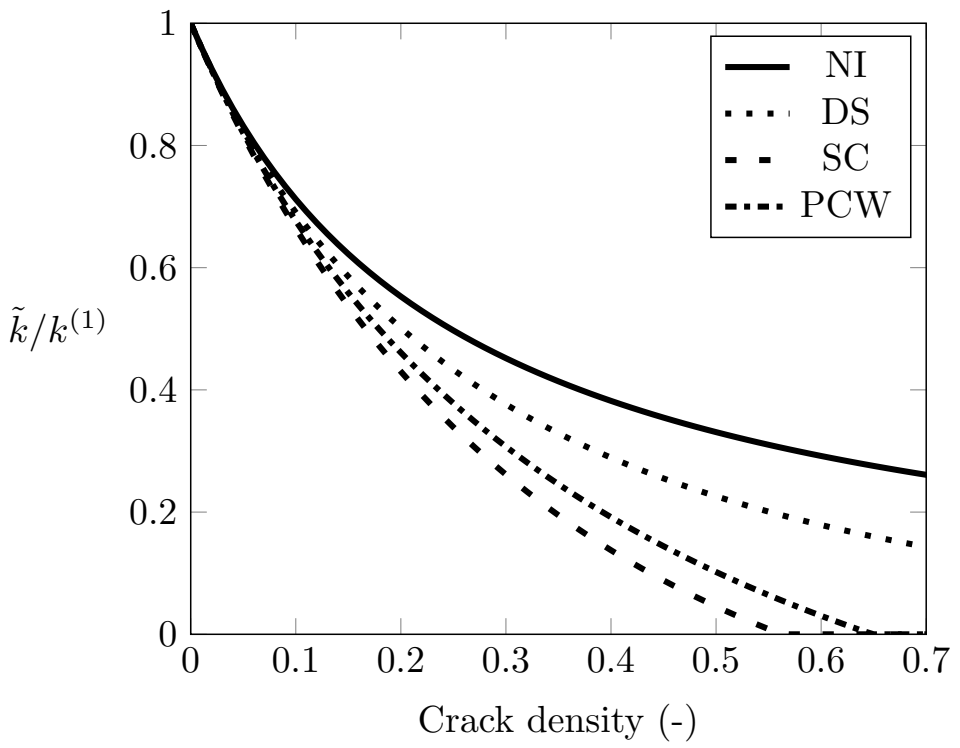

Figure 2: Normalized effective bulk modulus as a function of the crack density (for 3-D solids with $\nu^{(1)}=0.3$ ): comparison of the analytical results given by the non-interacting crack approximation (NI, continuous line), the differential scheme (Ds, loosely dotted curve), the self-consistent estimate (sc, loosely dashed line) and the PCW upper bound (PCW, dot-dashed line).

\subsection{Final remarks}

The PCW upper bound and several approximations were developed to estimate the effective conductivity and moduli of cracked media. Those presented in this section have been compared for an isotropic spatial distribution of isolated and randomly oriented ribbon-cracks $(d=2)$ and penny-shaped cracks $(d=3)$. Under these conditions, the non-interacting approximation seems to overestimate the effective conductivity or the moduli; for this reason, it will not be used hereafter. The self-consistent estimate and the PCW upper bound deliver consistent results.

Note that the above discussed estimates and bounds address microstructures in which cracks can be located in any position within the solid which will not be the case for the solids considered hereafter where cracks are prescribed to be located at inter-granular boundaries. In addition with these models, there are no explicit constraints, apart from global isotropy, on the spatial distribution of cracks with the exception of the PCW estimate which is a rigorous bound only for a specific class of microstructures as explained in section 2.2.1. As reported above, Sevostianov \& Kachanov (2019) recently proposed a model to derive the effective properties of a solid weakened by inter-granular cracks. This estimate as defined by relation (21) will be compared against full-field FFT computations in section 4, once its microstructural parameter $\rho_{s a t(d)}^{I G B}$ has been estimated for the synthetic microstructures 
considered in the next section 3. Moreover, a new estimate of the impact of inter-granular cracks on the effective conductivity is also developed in section 4 of this paper by modifying the PCW bound. This new estimate is compared to both relation (21) and the PCW upper bound defined by relation (11).

\section{Generation of synthetic microstructures}

The method used to generate synthetic microstructures representing uniformly weakened solids, as well as the method used to generate solids weakened at their inter-granular boundaries, are described in the following sections.

\subsection{Uniformly weakened solids}

Uniformly weakened 3-D microstructures are generated in two steps. First, $N_{l}$ seeds are randomly generated in the volume of interest $V$. These seeds are used as centers of disks with a radius $R_{l}$, representing the cracks. Each disk is itself inscribed in a sphere of the same radius $R_{l}$. In the rest of the article, these spheres are called "localization spheres" as they are used to "delimit" the crack location in order to control their spatial distribution (as explained in the following section). We introduce their density $d^{s p h}$ as the total volume of the spheres divided by $V$ :

$$
d^{s p h}=\frac{N_{l}\left(\frac{4}{3} \pi R_{l}^{3}\right)}{V}
$$

For 2-D microstructures, these seeds are the centers of the $N_{l}$ segments of length $2 R_{l}$, representing the $2-\mathrm{D}$ cracks, with each segment being inscribed in disks of radius $R_{l}$. The disks density $d^{d s k}$ is the total surface of the disks divided by $S$ :

$$
d^{d s k}=\frac{N_{l}\left(\pi R_{l}^{2}\right)}{S}
$$

Each crack orientation is chosen randomly for both 2-D and 3-D solids. In order to meet the constraint of periodicity, if a crack and its associated sphere in 3-D cases (and its associated disk in 2-D cases) intersect a boundary of the RVE (and RSE respectively), it is duplicated on the opposite face.

Two methods for generating these spheres can be used depending whether the cracks can overlap or not:

1. Non-overlapping cracks

The non-overlapping cracks are generated with a random sequential addition process (RSA, Torquato et al. (2006)). In $3-\mathrm{D}$ cases, $N_{l}$ seeds are generated sequentially to cover the volume $\overline{V \text { as }}$ long as possible with hard spheres of radius $R_{l}$. During the process, a seed location is modified and re-examined when the associated sphere of radius $R_{l}$ overlaps existing spheres until there is no more overlap. In 2-D cases, $N_{l}$ non-overlapping seed positions are obtained sequentially to cover the surface $S$ as long as possible with hard disks of diameter $2 R_{l}$. With this RSA process, the volume fraction of spheres coincides with their density $d^{s p h}$ and its maximal value attached to the 
RSA packing is approximately 0.38 (this limit is equal to 0.54 in $2-\mathrm{D}$ cases). At the end of this process, the solid is weakened by $N_{c}=N_{l}$ penny-shaped cracks of diameter $2 R_{l}$. It is worth remarking that the generated microstructures belong to the subclass of isotropic cracked media for which the PCW bound applies.

2. Overlapping cracks

Overlapping cracks are generated with a Poisson Boolean process. $N_{l}$ seed locations are added randomly and independently with respect to each other in a volume $V$ in $3-\mathrm{D}$ cases (and in a surface $S$ in 2-D cases) leading to possible overlaps of the associated spheres (or disks). In these conditions, the density of the overlapping localization spheres $d^{s p h}$ or disks $d^{d s k}$ deviates from the final concentration of the phase made of these spheres or disks denoted respectively $c^{s p h}$ or $c^{d s k}$. Indeed, these densities $d^{s p h}$ and $d^{d s k}$ can exceed $100 \%$. For a sufficiently large number $N_{l}$ of spheres (or disks in 2-D cases), the volume/surface fraction $c^{s p h}$ and $c^{d s k}$ can be well estimated by the theoretical expression relative to the Boolean model (see Lantuéjoul (2002) for more details):

$$
\begin{aligned}
c^{s p h} & =1-\exp \left(-\frac{4 \pi}{3} \frac{N_{l} R_{l}^{3}}{V}\right) \\
c^{d s k} & =1-\exp \left(-\frac{N_{l} \pi R_{l}^{2}}{S}\right)
\end{aligned}
$$

As full-field computations are performed using the FFT method (see further), cracks need to be given an thickness, denoted by $e$. This is performed in the second step of the microstructure generation. For a given disk defined by its position and its orientation, the Euclidean distance between the center of each voxel in the sphere and its projection on the disk in question is evaluated. A crack with a thickness $e$ is defined as the set of voxels in the overlapping sphere of radius $R_{l}$ whose center is at a distance to the disk of at most $e / 2$. In $2-\mathrm{D}$ cases, the Euclidean distance between each pixel in the localization disk and its projection on the segment, corresponding to the crack, is also used to assign a thickness $e$ to cracks.

The resulting microstructure can be described as a solid phase weakened by randomly distributed cracks of thickness $e$ and size $2 R_{l}$. As the generated cracks have a finite thickness, their volume fraction is nonzero. In the following, we only consider distributions of overlapping cracks when modeling the crack distribution in uniformly weakened solids. In these conditions and in the case where all cracks have the same thickness $e$ and size $2 R_{l}$, the volume fraction related to cracks $c^{(2)}$ can still be given by a relation similar to (24) by substituting the volume of the spheres by the one of the cracks because the obtained microstructure is a Boolean model of randomly oriented crack, so that:

$$
c^{(2)}=1-\exp \left(-\frac{N_{l} e \pi R_{l}^{2}}{V}\right)
$$

The number of localization spheres $N_{l}$ needed to reach a given crack density $\rho$ can then be estimated as follows:

$$
N_{l}=-\frac{V}{e \pi R_{l}^{2}} \ln \left(1-\pi \frac{e}{R_{l}} \rho\right)
$$


For a given crack density target $\rho$ and a choice of crack diameter $2 R_{l}$ and thickness $e$, equation (27) is used to provide the discrete number of localization seeds $N_{l}$. The microstructure is then generated using the overlapping crack scheme and $c^{(2)}$ is measured through a voxel count from which $A_{V}^{(2)}$ can be deduced (as $c^{(2)}=A_{V}^{(2)} e$ ). Then, the actual crack density of the generated microstructure, penny-shaped cracks of radius $l_{c}=2 R_{l}$, is computed from equation (5). Similar relations can of course be derived for 2-D cases.

Finally, let us note that, in this work, cracks are defined as planar disks in 3-D or as segments in 2-D. However, in the case of overlapping cracks, the crack geometry can be more complex and result from one or many disk or segment intersections. Hence, for instance, cracks with a polygonal line shape (in 2-D) can occur but their length will rarely exceed two or three segments. Also, without increasing the difficulty of the computations, the disks (or segments) inside the localization spheres could be replaced by more complex surfaces or curves like spherical shells or elliptical arcs.

\subsection{Solids weakened at their inter-granular boundaries}

The method for generating solids weakened at their inter-granular boundaries is composed of three steps which are presented below and illustrated in Figure 3.

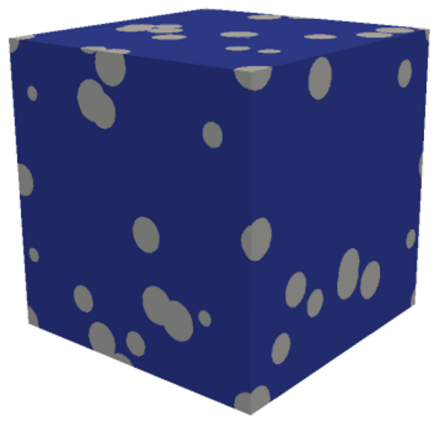

(a)

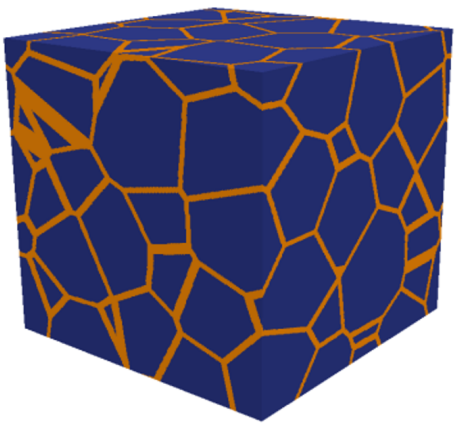

(b)

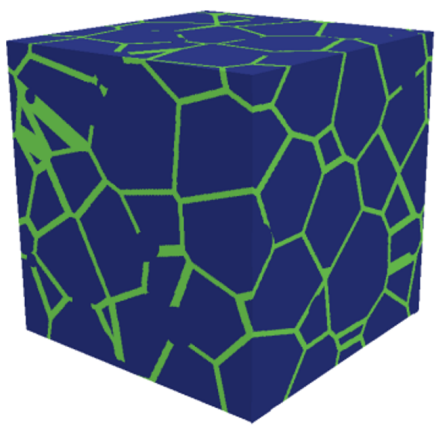

(c)

Figure 3: 3-D illustration of a solid weakened at inter-granular boundaries. Figure (a): Randomly distributed spheres (in grey) in a 3-D matrix (in blue). Figure (b): Grains (in blue) surrounded by an IGB phase (in orange). Figure 3(c): Example of 3-D microstructure obtained with the intersection of Figures (a) and (b) with a solid phase (in blue) and cracks located at inter-granular boundaries (in green).

The first step has already been described in section 3.1: the volume $V$ is packed with $N_{l}$ monodisperse spheres of radius $R_{l}$ using a Poisson Boolean process (see Figure 3(a)). In this manner, the generated spheres show a random distribution.

Second, a Voronoï tesselation with a desired monodisperse granulometric distribution is produced using an RSA process, which generates randomly-distributed seeds with mutual distances greater than or equal to $2 R$ in a cubic volume $V$ in $3-\mathrm{D}$ or a squared surface $S$ in 2-D of size $L$ (see Figure 3(b)). When the saturation limit associated with the RSA process is reached (i.e. 0.38 in $3-\mathrm{D}$ and 0.54 in $2-\mathrm{D}$, see section 3.1 ), the diameter of the targeted grains is close to $2 R$. The length $R$ and $L$ are chosen such that the grain size is much smaller 
than the size of the generated microstructure in order to obtain an RVE in 3-D or RSE in 2-D at the end of the process with a sufficiently large number of grains. In practice, the saturation limit reached at the end of this RSA process is very close to the theoretical one: 0.52 instead of 0.54 for 2 -D cases and 0.36 instead of 0.38 for 3 -D cases. The Voronoï cell walls are determined by finding each median plane between two neighboring seeds.

The obtained microstructure can then be considered as a two-phase solid composed of grains whose size is slightly larger than $2 R$ (since the saturation limit is not reached exactly) and surrounded by an inter-granular boundary phase. The proportion of the IGB phase in the medium is evaluated via its area per unit of volume $A_{V}^{(I G B)}$ (the equivalent in 2-D cases is the IGB length per unit of area $L_{A}^{(I G B)}$ ). Next, all the voxels which are at most $e / 2$ away from the Voronoï sides, are assigned to the IGB phase in order to generate microstructures with an IGB phase of thickness $e$. The volume fraction of the IGB phase can be estimated by $\left(A_{V}^{(I G B)} e\right)$. Note in Figure 3(b) that the IGB phase is totally interconnected.

In the final third step, solids weakened by cracks located at inter-granular boundaries can be generated in two different ways:

1. Cracks are defined as the intersection set between the localization spheres and the inter-granular boundaries. The rest of the inter-granular phase is allocated to the solid phase along with the grains in the volume. Hereinafter, this approach is referred to as the IGB approach.

2. The intersection of the localization spheres and the inter-granular boundaries is now defined as part of the solid phase along with the grains in the volume. The rest of the inter-granular phase is considered as cracks. As this approach is dual to the IGB approach, it is hereinafter referred to as the IGB-D approach.

Figure 3(c) shows the final IGB-D microstructure generated with the intersection of Figures 3(b) and 3(a). As shown in Figure 3(c), the developed numerical process makes it possible to generate interconnected crack networks distributed around the grains and randomly located. Periodic geometric conditions are applied to each step of the generation to ensure the periodicity of the final microstructure.

Note that a direct descriptor of the crack concentration is the crack area per unit of volume and is denoted by $A_{V}^{(2)}$. In 2 -D cases, the descriptor is $L_{A}^{(2)}$, the ribbon-crack length per unit of area. Once the crack sizes have been chosen conventionally (hereafter $l_{c}=2 R_{l}$ ), the crack density defined by relation (3) (relation (6) in 2-D cases) can also be used. An alternative way to quantify the crack concentration is the inter-granular coverage ratio $c_{c o v}^{(2)}$, defined in 3-D (and in 2-D respectively) as the ratio between the surfaces (or lengths) of the cracks and the IGB phase, namely $A_{V}^{(2)} / A_{V}^{(I G B)}$ (or $L_{A}^{(2)} / L_{A}^{(I G B)}$ in 2D-cases). In practice, these quantities are evaluated through voxel or pixel count taking into account crack or interface thickness $e$.

Finally, note that, whatever approach is used (IGB or IGB-D), the geometrical supports of the cracks are planes (in 3-D) and straight lines (in 2-D) since they are at inter-granular boundaries and the grains obtained by the Voronoï tessellation are convex polyhedra. More complex cracks geometrical supports could be obtained by using a different grain generation 
process such as, for example, the Johnson \& Mehl (1939) tessellation, which leads to grains that are not necessarily convex neither of polyhedral shape.

\subsection{Simulated microstructures and morphological ef- fects}

Table 1 shows the geometrical data used to generate the set of grains at the boundaries of which the cracks are distributed. The unit length in use is expressed in voxels or pixels and is denoted $U_{d}$. The parameters $R$ and $L$ as defined in previous sections are chosen to define the granular microstructures while $N$ is the resulting number of seeds after the saturation of the RSA process. The radius $R_{l}$ of the localization spheres (disks in 2-D cases) and the crack thickness $e$ are also reported. The choice of crack thickness $e$ is detailed in section 4.1.2.

\begin{tabular}{|c|c|c|c|c|c|c|c|c|}
\hline & $R$ & $N$ & $e$ & $L$ & $L_{A}^{(I G B)}$ & $A_{V}^{(I G B)}$ & $R_{l}$ & $\rho_{\text {sat }(d)}^{I G B}$ \\
\hline (units) & $\left(U_{d}\right)$ & $(-)$ & $\left(U_{d}\right)$ & $\left(U_{d}\right)$ & $\left(U_{d}^{-1}\right)$ & $\left(U_{d}^{-1}\right)$ & $\left(U_{d}\right)$ & $(-)$ \\
\hline 2-D & 50 & 157 & $\sqrt{2}$ & 1501 & 0.016 & - & 20 & 0.16 \\
\hline 3-D & 75 & 216 & $\sqrt{2}$ & 1000 & - & 0.017 & 30 & 0.16 \\
\hline
\end{tabular}

Table 1: Data used for the simulated microstructures (with $U_{d}=$ pixels in 2-D cases and $U_{d}=$ voxels in 3 -D cases)

The area per unit of volume of the inter-granular boundaries $A_{V}^{(I G B)}$ in 3-D or its equivalent length per unit of area $L_{A}^{(I G B)}$ in 2-D are also reported in Table 1. These microstructural parameters depend on the prescribed grain size. More systematic simulations of granular microstructures made it possible to establish that $L_{A}^{(I G B)}$ and $A_{V}^{(I G B)}$ scale well with $0.82 / R$ and $1.26 / R$ respectively (with $R$ expressed in $U_{d}, L_{A}^{(I G B)}$ and $A_{V}^{(I G B)}$ in $U_{d}^{-1}$, see Table 1 ). The mean deviation between these correlations and the simulated data tends to be less than $1 \%$ for a radius $R$ varying from $15 U_{d}$ to $100 U_{d}$. For the following simulations, the radius $R$ has been fixed to the values reported in Table 1 for 2-D and 3-D solids.

Moreover, whatever the choice of initial positions for the Voronoï seeds, only variations up to the fifth decimal place were observed on $A_{V}^{(I G B)}$ (and $L_{A}^{(I G B)}$ respectively) which can be considered as negligible. Accordingly, the positions of Voronoï seeds for each type of synthetic microstructures will be kept fixed in the following simulations; this makes it easier to study the impact of crack location on the effective properties.

With the data reported in Table 1, Figure 4 shows the correlations between the intergranular coverage ratio and the density of localization spheres for the 3-D IGB and IGB-D microstructures generated. Note that, for a given density of localization spheres, the intergranular coverage ratio $c_{c o v}^{(2)}$ is well estimated by the volume fraction of spheres $c^{s p h}$ as given by relation (24) for the IGB approach and by $\left(1-c^{s p h}\right)$ for the IGB-D approach which suggests that the number of the localization spheres in the studied volume $V$ is large enough. It is then possible to quite accurately determine the volume fraction of cracks by multiplying the inter-granular coverage ratio by the volume fraction of the IGB phase. For 2-D solids, similar results were obtained and showed that the inter-granular coverage ratio $c_{\text {cov }}^{(2)}$ can be well approximated by $c^{d s k}(\mathrm{IGB})$ or $\left(1-c^{d s k}\right)$ (IGB-D), with $c^{d s k}$ being defined by relation 


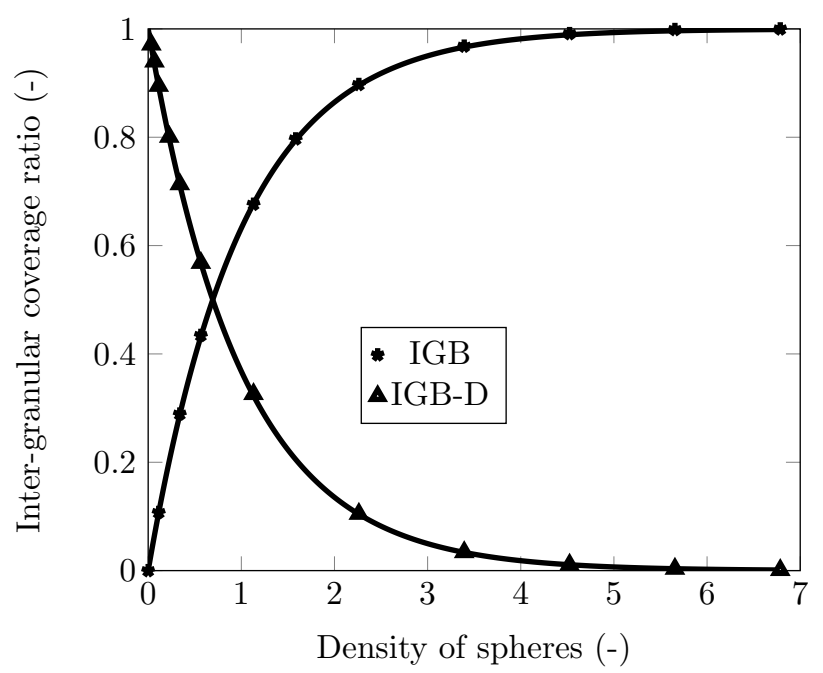

Figure 4: Comparison of the the simulated inter-granular coverage ratio $c_{c o v}^{(2)}$ for IGB and IGB-D 3-D microstructures (circles and triangles respectively) with analytical expressions in straight lines $\left(\left(1-e^{-\frac{4 \pi}{3} \frac{N_{l} R_{l}{ }^{3}}{V}}\right)\right.$ and $e^{-\frac{4 \pi}{3} \frac{N_{l} R_{l}{ }^{3}}{V}}$ for respectively each approach)

As explained above, the crack density herein has been defined with the convention $l_{c}=$ $2 R_{l}$. Thus, once $R_{l}$ and the size of the RVE fixed, the crack density only depends on the number $N_{l}$ of localization spheres (or disks in 2-D cases) which can be expressed as a function of $A_{V}^{(2)}\left(=A_{V}^{(I G B)} c_{\text {cov }}^{(2)}\right.$ using relation (4)) or $L_{A}^{(2)}$ in 2-D (see section 2.1), namely:

$$
\rho=\frac{R_{l}}{\pi} A_{V}^{(I G B)} c_{\text {cov }}^{(2)} \quad(\text { in 3-D cases }) \quad \text { and } \quad \rho=\frac{R_{l}}{2} L_{A}^{(I G B)} c_{\text {cov }}^{(2)} \quad \text { (in 2-D cases) }
$$

Furthermore, the crack density at saturation, denoted by $\rho_{\text {sat }(d)}^{I G B}$, corresponding to the case where all the inter-granular boundaries are covered by cracks (i.e. $\left.c_{\text {cov }}^{(2)}=1\right)$ is given by:

$$
\rho_{\text {sat }(3)}^{(I G B)}=\frac{R_{l}}{\pi} A_{V}^{(I G B)} \quad \text { (in 3-D cases) } \quad \text { and } \quad \rho_{\text {sat }(2)}^{(I G B)}=\frac{R_{l}}{2} L_{A}^{(I G B)} \quad \text { (in 2-D cases) }
$$

We chose $R_{l}$ in $2-\mathrm{D}$ or 3 -D cases in order to obtain the same crack density at saturation, namely $\rho_{\text {sat }(2)}^{I G B}=\rho_{\text {sat }(3)}^{I G B} \approx 0.16$ (see Table 1$)$.

For a given crack density $(\rho \approx 0.05)$ lower than its saturation value, Figure 5 shows an example of two 2-D microstructures with the same ribbon-crack density obtained by simulation: a solid weakened at their inter-granular boundaries (left) and a uniformly weakened solid (right). For both simulated microstructures, cracks can overlap but as expected, crack coalescence seems more pronounced as shown in Figure 5(a) when cracks are located at inter-granular boundaries. We show further on that this point plays a key role in the effective properties.

To understand the influence of the radius $R_{l}$ of the localization spheres (or disks in 2-D cases) when the grain size $2 R$ is kept fixed, we considered two extreme situations corresponding to a very high or low $R / R_{l}$ ratio. Figure 6 depicts two 2-D microstructures obtained this way. 


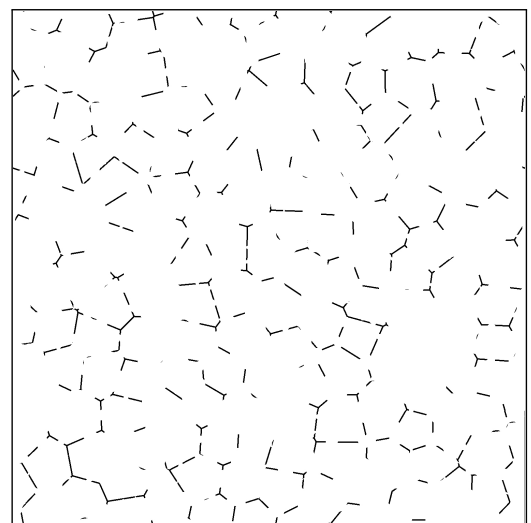

(a)

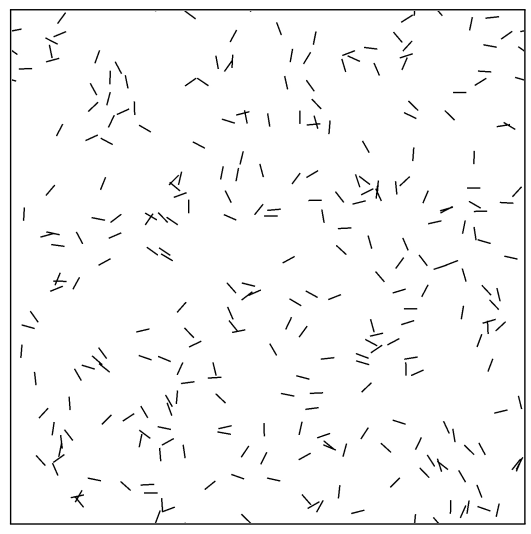

(b)

Figure 5: Two random distributions of ribbon-cracks for a crack density of $\rho \approx 0.05$. Figure (a): Cracks located at inter-granular boundaries. Figure (b): Cracks dispersed over the whole surface. The cracks thickness has been enlarged to $e=4$ to enhance the contrast of these images.

On the one hand, when the ratio is high $\left(R / R_{l} \gg 1\right.$, Figure $\left.6(\mathrm{a})\right)$, the localization spheres in 3-D cases (or disks in 2-D cases) are small compared with the grain size. In this way, the cracks are randomly distributed along inter-granular boundaries and are not connected. On the other hand, when this ratio is low $\left(R / R_{l} \ll 1\right.$, Figure $\left.6(\mathrm{~b})\right)$, the cracks are spatially located in clusters whose centers and radii are given by those of the spheres in 3-D or disks in 2-D. In these spherical zones (circular for 2-D solids), the grains are entirely isolated which means these areas can be assimilated to perfectly insulating spherical (or circular) clusters. Therefore, by adjusting this ratio $\left(R / R_{l}\right)$, we can simulate very different types of crack morphologies located on inter-granular boundaries.

For most of the simulated microstructures studied in section 4 , the ratio $R / R_{l}$ is set to 2.5 (see data in Table 1 ). The effect of this ratio on the effective conductivity is specifically studied in section 4.3 .

\section{Effect of the spatial distribution of cracks on the ef- fective thermal conductivity of solids}

Let us now consider the RVE of a solid weakened by cracks and evaluate its effective thermal conductivity by full-field computations based on the FFT method that is briefly introduced in section 4.1. In particular, the choice of the ribbon-crack thickness is explained in this section. The impact of the cracks density and spatial distribution on the effective conductivity is studied in section 4.2 , the crack density being determined by the number $N_{l}$ of localization spheres (or disks in 2-D) as explained in section 3.3. In section 4.3, the impact of morphological effects on IGB microstructures is studied by modifying the radius $R_{l}$ of the localization disks. Apart from this modification of $R_{l}$ in this section, all characteristics of the considered IGB microstructures are fixed and are those reported in Table 1 above. 


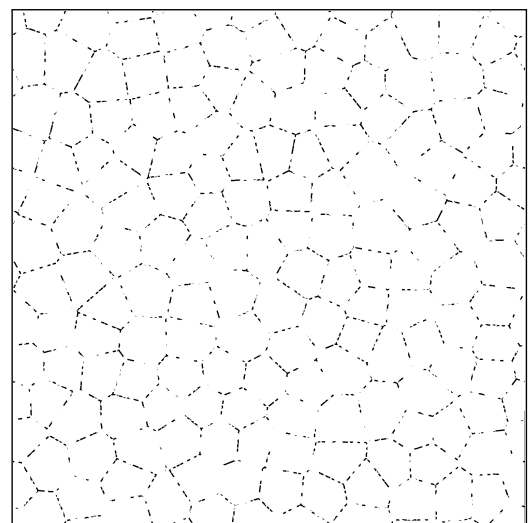

(a)

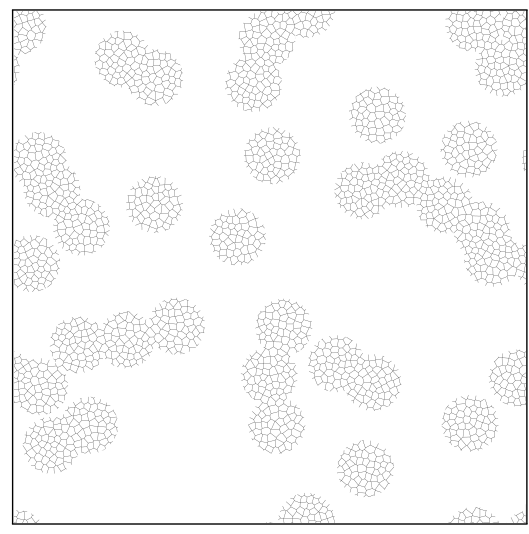

(b)

Figure 6: Two extreme 2-D cases in terms of their morphology for the same surface fraction of localization disks $(0.3)$ and the same inter-granular coverage ratio $\left(c_{\text {cov }}^{(2)}=0.3\right)$. Figure (a): $R / R_{l}=10$, cracks are randomly distributed along the whole inter-granular boundaries of the solid. Figure (b): $R / R_{l}=0.1$, cracks are still inter-granular but now located in preferential zones (clusters) corresponding to the localization disks. The cracks thickness is enlarged to $e=4$ to enhance the contrast of these images.

\subsection{Full-field computations}

\subsubsection{FFT resolution}

In steady-state conditions with no source terms, the thermal field $T(\boldsymbol{x})$ and the heat flux field $\boldsymbol{j}(\boldsymbol{x})$ are solutions of $(\boldsymbol{x} \in V)$ :

$$
\nabla \cdot \boldsymbol{j}(\boldsymbol{x})=\mathbf{0} \quad \text { with } \quad \boldsymbol{j}(\boldsymbol{x})=-\sigma(\boldsymbol{x})(\nabla T(\boldsymbol{x}))
$$

where $\nabla$ is the gradient operator. For an isotropically cracked solid, the effective thermal conductivity is a scalar denoted by $\tilde{\sigma}$ and defined as:

$$
\langle\boldsymbol{j}\rangle=-\tilde{\sigma}\langle\nabla T\rangle
$$

where $\langle$.$\rangle denotes the volume average over the RVE V$.

In order to solve the boundary value problem (relation (30)), we used the fast Fourier transform (FFT) method originally proposed by Moulinec \& Suquet (1998). Periodic boundary conditions were considered to solve (30) and the macroscopic temperature gradient is prescribed on average over the volume $V$. The considered RVE $V$ in 3-D cases (RSE $S$ in 2-D cases) is discretized on a regular grid with a spatial resolution of 1 length unit/voxel (1 length unit/pixel). An Anderson extrapolation method (see Anderson (1965); Toth et al. (2015); Ramière \& Helfer (2015)) was also used in the FFT based algorithm to increase the convergence rate with a storage depth parameter of 5 . The convergence ratio is taken as $10^{-5}$. For $2-\mathrm{D}$ and 3 -D cases, the number of pixels and voxels are about $\left(2 \times 10^{6}\right)$ and $10^{9}$, respectively.

The work detailed in Gasnier et al. (2018) shows that the use of FFT filters can improve the numerical results and best results can be expected with the backward-and-forward finite-

difference Fourier scheme (BF-FFT, Willot \& Pellegrini (2008)). In this scheme, the flux and temperature gradient fields are known along a discrete set of voxel corners in 3-D (or pixel corners in 2-D). Based on Gasnier et al. (2018) the efficiency of the BF-FFT filter is 
evaluated in the section below while estimating the necessary crack thickness for the FFT resolution of cracked microstructures.

\subsubsection{Choice of crack thickness}

In line with Gasnier et al. (2018), Figure 7 shows the effect of the crack thickness on the apparent thermal conductivity $\frac{1}{c^{(1)}} \frac{\tilde{\sigma}}{\sigma^{(1)}}$. The contrast ratio is $\sigma^{(2)} / \sigma^{(1)}=10^{-4}$ for the results reported in this Figure. The effect of the BF-FFT scheme Willot \& Pellegrini (2008) is also studied in this Figure. Note that the correction $\left(1 / c^{(1)}\right)$ related to the apparent thermal conductivity is consistent with the limit where the cracks have no volume in $3-\mathrm{D}$ cases and no surface in $2-\mathrm{D}$ cases, i.e. $c^{(1)}=1$.

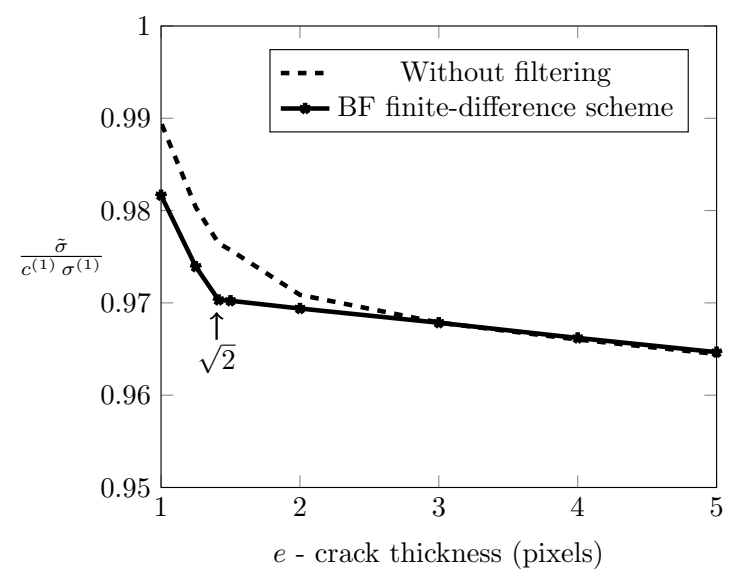

(a)

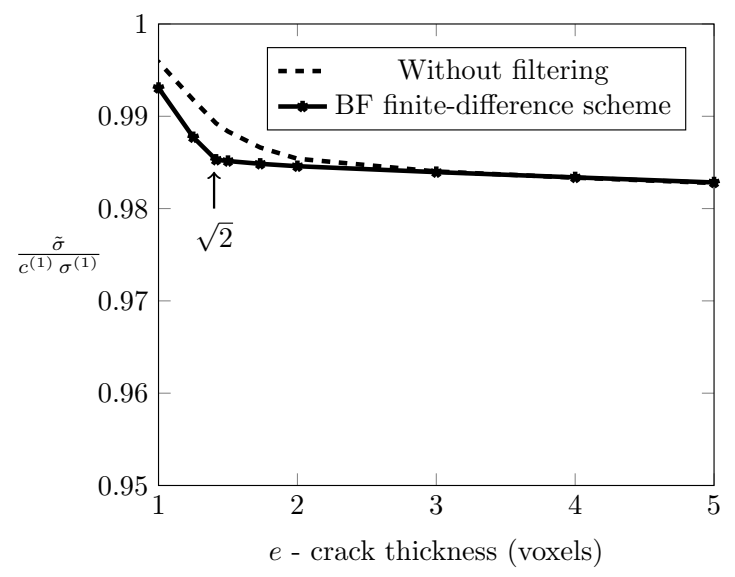

(b)

Figure 7: Influence of the crack thickness on the normalized apparent conductivity of the IGB microstructure and comparison between the basic FFT and the BF-FFT schemes. Figure (a): 2-D case with $N_{l}=200$. Figure (b): 3 -D case with $N_{l}=900$.

As shown in Figure 7 when the crack thickness equals one voxel in 3-D cases (and one pixel in 2-D cases) the results clearly differ from those obtained with larger values. For 2-D cases and a crack thickness larger than $\sqrt{2}$ pixels, a linear trend is observed for FFT estimates as derived with the BF-FFT scheme (Figure 7(a)). The same linear trend is also observed with FFT estimates derived without filtering but in a much smaller interval (only for a crack thickness larger than 3 pixels $)$. This linear trend reads: $\frac{1}{c^{(1)}} \frac{\tilde{\sigma}}{\sigma^{(1)}} \approx-\left(3.2 \times 10^{-3}\right) e+0.97$. The use of the BF-FFT scheme enables the generation of the thinnest cracks with a better estimation of the y-intercept of the regression line as the interpolation interval is wider: we obtain $\tilde{\sigma} / \sigma^{(1)} \approx 0.97$ at $e=0\left(c^{(1)}=1\right)$. This extrapolated value is close to that given for $e=\sqrt{2}$ pixels and the BF-FFT scheme.

A similar sensitivity study is reported in Figure 7(b) for 3-D cases and the same contrast ratio. Here, the number of localization spheres $N_{l}$ equals 900 . The reported trends are similar to that resulting for the 2-D case in Figure 7 (a) with a change in slope at $\sqrt{2}$ voxels with the BF-FFT scheme. It is worth pointing out that a crack thickness of 1.5 voxels (close to $\sqrt{2}$ ) for the change in slope was chosen by Gasnier et al. (2018) for randomly weakened solids by penny-shaped cracks. These results are then consistent with Gasnier et al. (2018). With a crack thickness greater than or equal to $\sqrt{2}$ (as 1.5), the generated cracks are thick enough 
to be considered as thermal barriers in FFT.

Based on these results, the BF-FFT scheme and a crack thickness $e=\sqrt{2}$ will be used in the following 2-D and 3-D FFT computations.

\subsection{Effect of the spatial distribution of perfectly insu- lated cracks on the effective conductivity}

\subsubsection{Contrast effects at saturation}

We investigated the saturation limit for cracks distributed along inter-granular boundaries. The saturation limit is reached for an inter-granular coverage ratio $c_{\text {cov }}^{(2)}$ of $100 \%$. At this limit, the generated microstructure is no longer a solid but a granular medium.

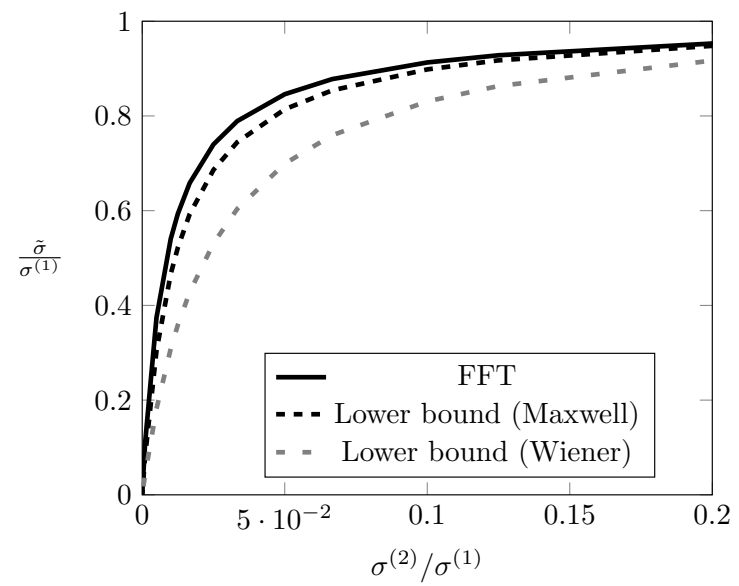

(a)

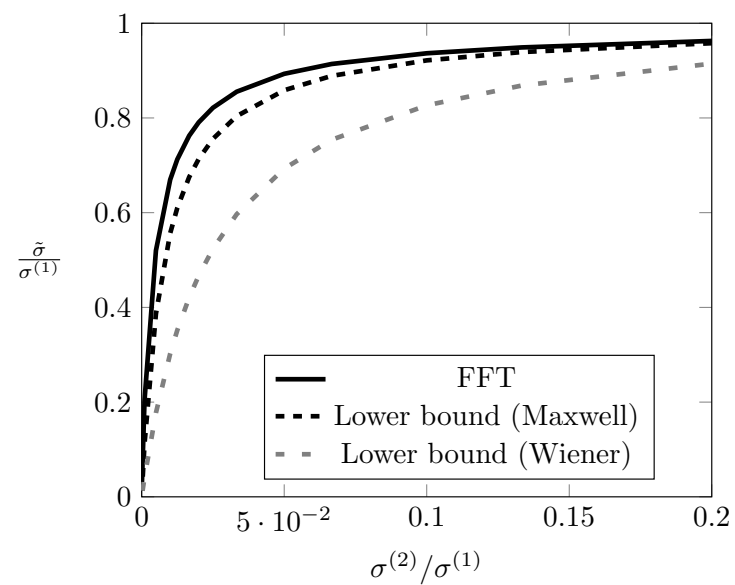

(b)

Figure 8: Normalized effective conductivity as a function of the contrast ratio between phases for the solid weakened by cracks covering the whole IGB phase. Figure (a): the 2-D case. Figure (b): the 3-D case.

Concerning first the 2-D solid, changes in the normalized effective conductivity with the contrast ratio $\left(\sigma^{(2)} / \sigma^{(1)}\right)$ are reported in Figure 8(a). As expected, the normalized effective conductivity tends to zero when the contrast ratio tends to zero as the grains are perfectly insulated by the connected network of cracks. Concerning the 3 -D medium, the results are reported in Figure 8(b) and show similar trends.

Figure 8 also shows the two lower bounds, i.e. the Wiener lower bound (relation (2)) and the Maxwell lower bound (relation (1)). Remarkably, the Maxwell lower bound yields results slightly lower than those given by FFT computations.

To obtain a normalized effective conductivity lower than $\left(5 \times 10^{-3}\right)$, the contrast ratio has to be at least equal to $10^{-4}$ for both studied cases. For this reason, we decided to adopt a very low but non-zero contrast ratio to model fully insulating cracks.

\subsubsection{Impact of the crack density on the thermal conductivity of the different microstructures}

Figures 9(a) and 9(b) show the evolutions of the normalized effective conductivity $\left(\tilde{\sigma} / \sigma^{(1)}\right)$ with the crack density of perfectly insulating cracks for 2-D and 3-D cases respectively. The 
results for solids weakened by uniformly distributed cracks (U) differ significantly from those related to solids weakened at their inter-granular boundaries (IGB). These results are also compared with the PCW upper bound (relation (11)) in Figures 9, right.

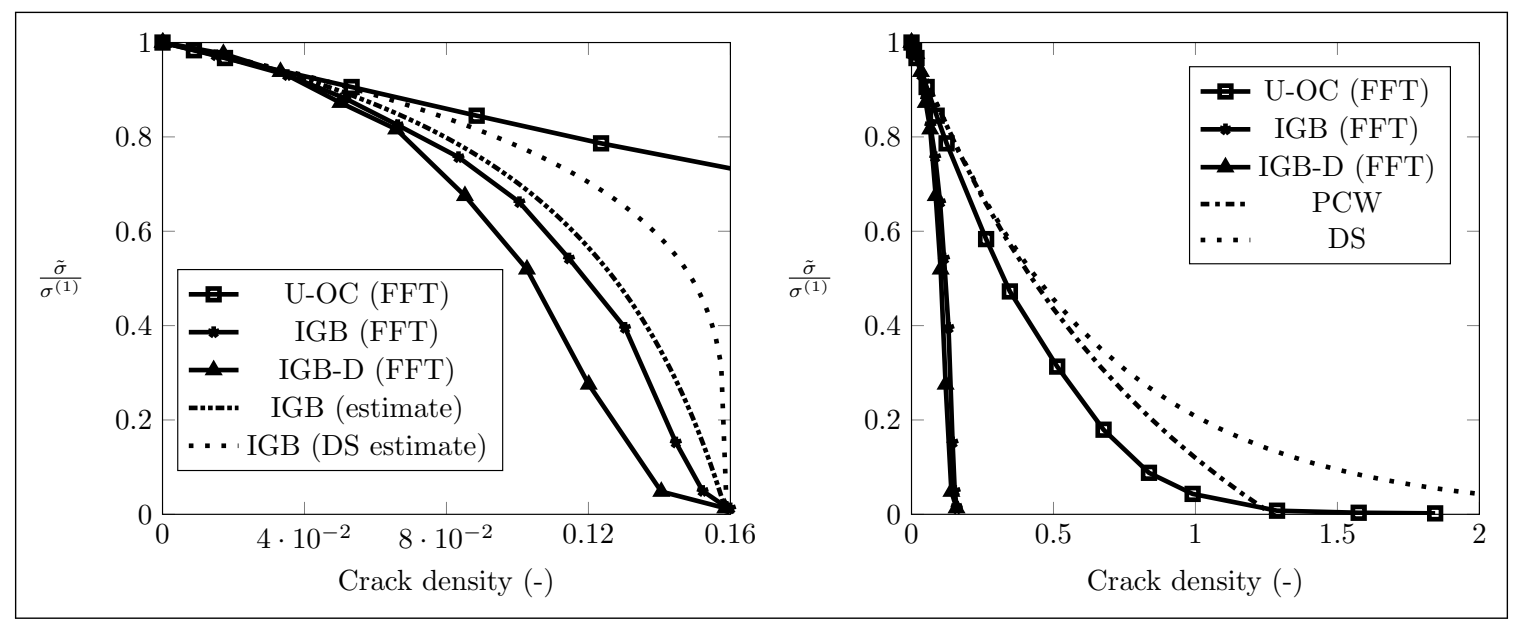

(a)

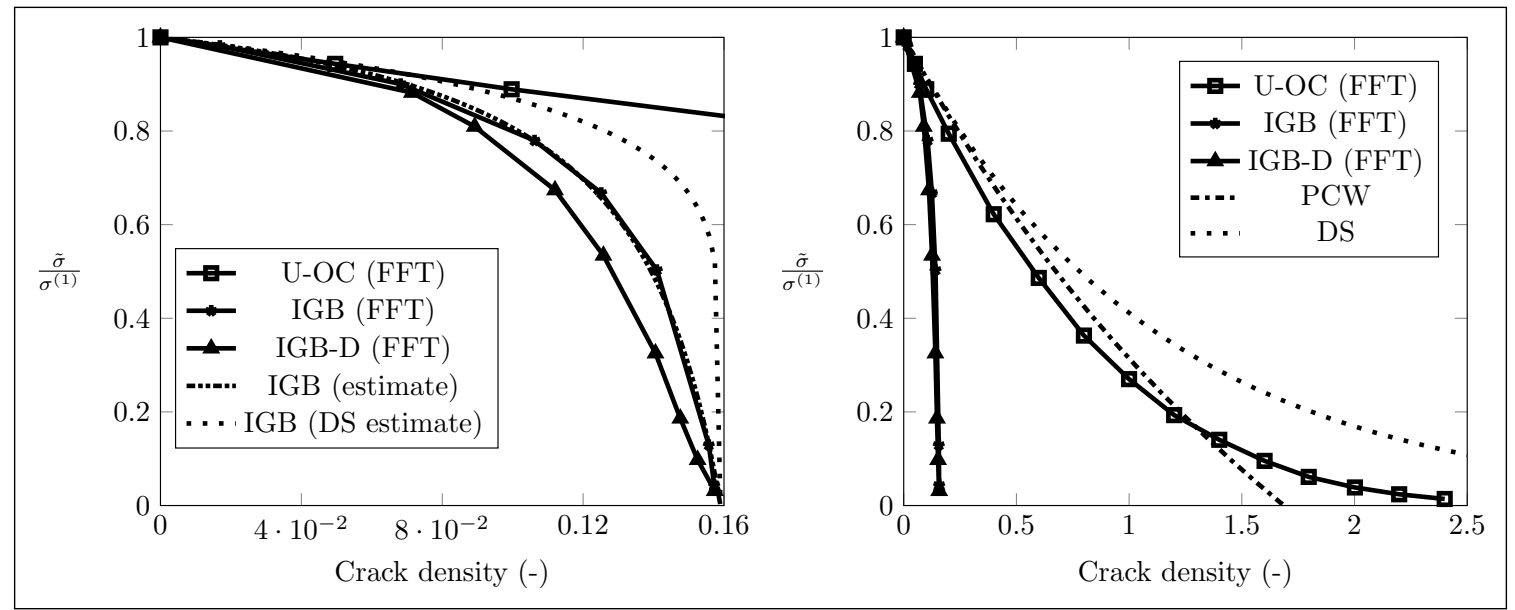

(b)

Figure 9: Normalized effective conductivity as a function of the crack density in 2-D cases (Figure (a)) and in 3-D cases (Figure (b)). FFT computations (solid lines) for different crack distributions: uniformly distributed overlapping cracks "U-OC" and cracks located at inter-granular boundaries with both "IGB" and "IGB-D" approaches. Left figure: zoom on the interval $[0 ; 0.16]$ of crack density, FFT results are compared with the "DS estimate" derived by Sevostianov \& Kachanov (2019) (loosely dotted line) and with the new "IGB" estimate (dot-dashed line) defined by relation (32). Right figure: overall evolution, FFT results are compared with the PCW upper bound (PCW, dot-dashed line) and the differential scheme (DS, loosely dotted line).

For 2-D and 3-D uniformly weakened cases with overlapping cracks (U), the normalized effective conductivity is close to that predicted with the PCW upper bound (PCW) at low crack density despite the fact that a non-zero crack thickness is adopted. This result once again underlines that the fixed crack thickness $e=\sqrt{2}$ is small enough to ensure a relevant crack representation for the FFT computations. For larger crack densities, FFT results related to uniformly weakened solids deviate slightly from those of the PCW upper bound. As explained in section 2.2, this bound is only an estimate for crack densities larger than 0.24 
in 3-D (and 0.32 in 2-D). Despite this limitation, we observe that the PCW estimate delivers predictions that are consistent yet slightly higher than the FFT ones for uniformly weakened 2-D solids. For uniformly weakened 3-D solids, the PCW estimate fits fairly well FFT results even for large crack density values. However, it yields a lower cut-off crack density (approximately 1.4 and 1.6 for 2-D and 3-D cases respectively). The fact that the differential scheme underestimates the effect of the crack density on the effective conductivity with respect to the PCW predictions suggests that cracks interactions are apparently better captured with the PCW approach even for the limit situation of overlapping cracks. This might derive from the construction of the DS model in which cracks interactions are essentially modeled by an isolated crack surrounded by an infinite medium while in the Hashin-Shtrikman approach on which PCW bounds rely, pair-wise interactions are more explicitly accounted for.

The drop of the effective conductivity of microstructures weakened at their inter-granular boundaries occurs at much lower crack density values than for uniformly weakened ones: $\rho_{s a t(d)}^{I G B} \approx 0.16$ for both IGB and IGB-D microstructures in 2-D and 3-D (see Table 1).

As suggested in section 3.3, the crack distribution along inter-granular boundaries leads to fewer crack locations possibilities which induces crack interactions at lower crack densities compared with solids with uniformly distributed cracks. Of course, both IGB and IGB-D approaches coincide in the two limiting conditions, i.e. $\rho=0$ (no cracks) and $\rho=\rho_{\text {sat }(d)}^{I G B}$ (cracks covering the whole IGB phase). However, as the IGB-D microstructure displays connected cracks along the inter-granular boundaries (because localization spheres in 3-D or disks in 2-D yield isolated thermal bridges in this type of microstructures), the corresponding effective conductivity is lower than the IGB conductivity (in which the localization spheres in 3-D or disks in 2-D yield isolated thermal insulators).

\subsubsection{A new estimate of the effective conductivity for solids weakened at IGB inferred by full-field simulated results}

Once the radius of localization spheres in 3-D cases (or disks 2-D cases) has been arbitrarily fixed, the cut-off crack density only depends on the spatial distribution of cracks. Therefore, the expression of the upper bound (11) can be used to provide new relevant estimates with the following constraints:

- the slope at $\rho=0$ coincides with the non-interacting crack model one $\left(=-\beta_{d}\right)$;

- the effective conductivity vanishes when the crack density tends to the cut-off crack density, this last constraint depending on the considered microstructure.

Therefore, the new estimate for the IGB and IGB-D microstructures is given by substituting $\rho_{(d)}^{\mathrm{PCW}+}$ by $\rho_{s a t(d)}^{I G B}$ in the expression of the upper PCW bound (relation (11)):

$$
\tilde{\sigma}^{\mathrm{IGB}}(\rho)=\sigma^{(1)}\left(\frac{1-\rho / \rho_{\text {sat }(d)}^{I G B}}{1+\left(\beta_{d}-1 / \rho_{\text {sat }(d)}^{I G B}\right) \rho}\right)
$$

For the considered 2-D and 3-D microstructures of solids weakened at their inter-granular boundaries, we have already explained (see section 3.3) that the sizes of the localization spheres for 3-D cases (and disks for 2-D cases) were chosen so that the crack density at 
saturation $\rho_{\text {sat }(d)}^{I G B}$ does not depend on the spatial dimension $(d)$ (here, $\rho_{\text {sat }}^{I G B} \approx 0.16$, see Table 1). The results of this new estimate are close to those given by the FFT computations for 2-D (Figure 9(a), left) and 3-D cases (Figure 9(b), left)). Remarkably, the FFT results for solids weakened at their inter-granular boundaries simulated with the IGB approach are well estimated with the new IGB estimate with a quasi overlap of both curves in 3-D (see Figure $9(\mathrm{~b})$ ). However, the effective properties of the solids weakened with the IGB-D approach remain over-predicted by the proposed estimate.

The predictions of the model proposed recently by Sevostianov \& Kachanov (2019) to estimate the effective conductivity of solids weakened at their inter-granular boundaries (see relation (21), section 2.4) have been reported for 2-D (see Figure 9(a), left) and 3-D cases (see Figure 9(b), left). As this last model also complies with the two constraints defined above, the initial slope and the cut-off crack density are as expected perfectly estimated. However, the drop in the effective conductivity seems to be under-predicted by this model. Therefore and at least for the microstructures considered herein, the estimates derived from the PCW upper bound give more accurate results than those provided by the model derived from the differential approximation and proposed by Sevostianov \& Kachanov (2019). For this reason, the only estimate considered in the following for solids weakened at their intergranular boundaries is the one proposed in this paper and defined by relation (32).

\subsection{Morphological effects}

Hereinafter, only the IGB approach is considered to model solids weakened at their intergranular boundaries. As explained in section 3.3, different microstructures can be generated by adjusting the grain size $R$ to the localization disk radius $R_{l}$ ratio, $R / R_{l}$. These microstructures range from crack clusters uniformly distributed in the solid $\left(R / R_{l} \ll 1\right)$ to un-connected small cracks randomly distributed along the IGB phase $\left(R / R_{l} \gg 1\right)$. Figure 10 shows the influence of this ratio on the normalized effective conductivity of the studied 2-D and 3-D cases.

Note that all the curves in the Figure 10 (for a given $d$-case) converge at the same values $\left(\tilde{\sigma} / \sigma^{(1)}\right)$ for the two extremes (no cracks along inter-granular boundaries $-c_{c o v}^{(2)}=0$ - and cracks covering the whole IGB $\left.-c_{\text {cov }}^{(2)}=1\right)$. .

As explained in section 3.3, when the ratio of radii is high $\left(R / R_{l} \gg 1\right)$, the cracks are randomly distributed along inter-granular boundaries and are not connected (see the example of RSE reported in Figure 6(a)). As a result, the heat flux can pass through the inter-granular boundaries which leads to the highest possible value of the effective conductivity for a given inter-granular coverage ratio. In the limit $\left(R / R_{l} \gg 1\right)$, inter-granular boundaries can be viewed as homogeneous at the grain scale but heterogeneous at a much smaller scale related to the cracks size so that two homogenization models can be applied at two separated scales. At the smallest scale, the effective conductivity of the inter-granular boundaries is estimated as a function of the given inter-granular coverage ratio $c_{c o v}^{(2)}$ with the help of specific unit-cell FFT computations. Once this effective conductivity known, previous results (Figure 8) can be used to estimate the effective conductivity of the solid at the largest scale. More details are given about this specific model in Appendix A. This upper estimate is reported in Figure 10 for 2-D and 3-D cases. For 2-D cases, this estimate delivers consistent predictions when compared with FFT results related to the largest ratio $R / R_{l}=10$. 


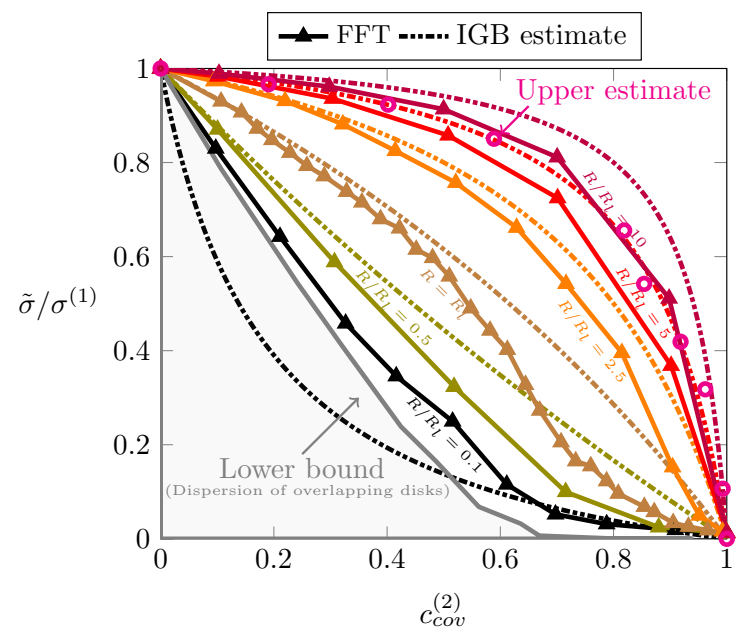

(a)

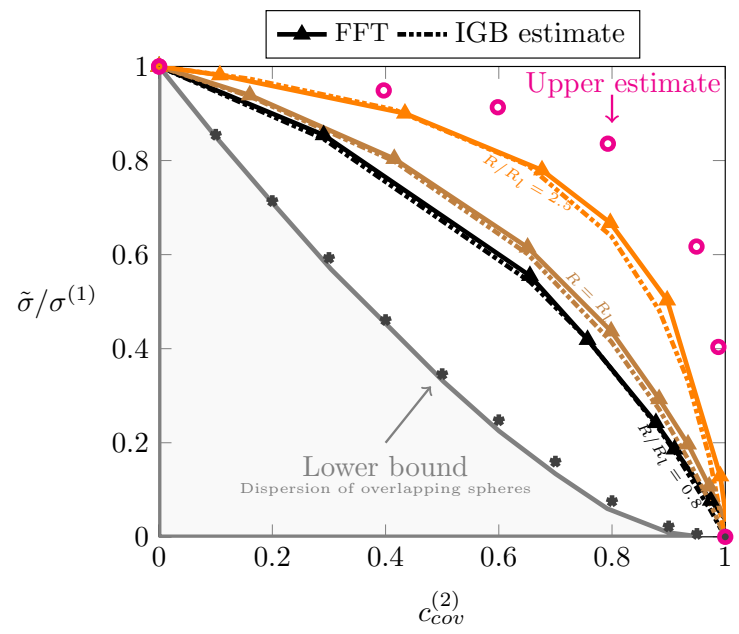

(b)

Figure 10: Normalized effective conductivity as a function of the inter-granular coverage ratio in 2-D (Figure (a)) and 3-D cases (Figure (b)) weakened at the IGB: effect of the $R / R_{l}$ ratio (FFT computations indicated in solid lines and dashed line for the IGB estimate, star-shaped dots for anterior results obtained by Kim \& Torquato (1992)), open pink circles for the upper estimates defined in Appendix A.

On the other hand, when this ratio of radii is low $\left(R / R_{l} \ll 1\right)$, the cracks are spatially located in circular zones where grains are entirely isolated. Therefore, these areas can be assimilated to perfectly insulating circular clusters (see an example of RSE Figure 6(b)). These isolated voids significantly decrease the effective conductivity so that, for a given inter-granular coverage ratio, this type of microstructures shows lower effective conductivity compared with microstructures with no crack clusters. The limit $R / R_{l} \rightarrow 0$ corresponds to a medium weakened by a dispersion of overlapping voided spheres or disks in 2-D (the inter-granular coverage ratio being respectively the volume fraction or the surface fraction of the voids). This lower bound has been computed by FFT and is reported in Figure 10.

Between these two extreme types of microstructures with regard to their morphology for a given inter-granular coverage ratio, the effective conductivity is an increasing function of the $R / R_{l}$ ratio.

Note that, as $c_{c o v}^{(2)}=\rho / \rho_{\text {sat }(d)}^{I G B}$, the estimate (32) can be easily expressed as a function of the inter-granular coverage ratio $c_{c o v}^{(2)}$, namely:

$$
\tilde{\sigma}^{\mathrm{IGB}}\left(c_{c o v}^{(2)}\right)=\sigma^{(1)}\left(\frac{1-c_{c o v}^{(2)}}{1+\left(\beta_{d} \rho_{s a t(d)}^{I G B}-1\right) c_{c o v}^{(2)}}\right)
$$

As a result, the slope of this curve near $c_{\text {cov }}^{(2)}=0$ equals $\left(-\beta_{d} \rho_{\text {sat }(d)}^{I G B}\right)$ and, as the crack density at saturation depends on $R_{l}$ (relation (29)), this slope also depends on $R_{l}$ (with $R$ and $L_{A}^{(I G B)}$ kept fixed for these simulations as indicated above). For 2-D computations (as shown in Figure 10(a)), this initial slope as well as the decrease of the effective conductivity with the coverage ratio are well captured by the estimate (32) at least when the $R / R_{l}$ ratio is within $[0.5 ; 2]$. This is no longer the case for lower or larger values of this ratio. For 
instance, the initial slope as predicted by full-field computations is no longer consistent with that corresponding to the dilute dispersion of cracks in 2-D cases as the cracks are either concentrated in clusters $\left(R / R_{l} \ll 1\right)$ or at inter-granular boundaries $\left(R / R_{l} \gg 1\right)$. Therefore, estimate (32) significantly deviates from full-field results when the $R / R_{l}$ ratio equals 0.1 or 10 as in the cases of the 2-D microstructures shown in Figure 6.

Due to computational constraints, the effect of the ratio $R / R_{l}$ on the effective conductivity has been less extensively studied for 3 -D cases. Figure 10(b), however, shows the evolution of the effective conductivity as a function of the inter-granular coverage ratio of perfectly insulating cracks for different $R / R_{l}$ values ranging from 0.8 to 2.5 (remember that $R / R_{l}=2.5$ is the ratio considered in previous computations, e.g. Figure $9(\mathrm{~b})$ ). Consistently with the $2-\mathrm{D}$ results and as expected, the effective conductivity does not depend on the $R / R_{l}$ ratio for the two limit values of the inter-granular coverage ratio: no cracks along inter-granular boundaries $\left(c_{c o v}^{(2)}=0\right)$ and entirely voided inter-granular boundaries $\left(c_{c o v}^{(2)}=1\right)$. In addition, the conclusions drawn from 2-D computations remain true: for a given inter-granular coverage ratio $c_{\text {cov }}^{(2)}$, the effective conductivity is still an increasing function of the $R / R_{l}$ ratio and lies above results related to a solid weakened by overlapping perfectly insulating spheres (continuous line). It is worth remarking that these last results are very close to past results obtained by Brownian motion simulation (Kim \& Torquato (1992)) for the random distribution of overlapping spheres (star-shaped dots). Finally, the effective conductivity computed by FFT computations lies below the upper estimate defined in Appendix A and reported in Figure 10(b) (open pink circles).

\section{$5 \quad$ New bounds and estimates of the elastic properties of an isotropic solid weakened by micro-cracks located at inter-granular boundaries}

The estimate defined by relation (32) can be directly used to compute the effective dielectric constant or the magnetic permeability or even the diffusion coefficient by substituting the conductivity of the two phases by one of these physical properties. Considering the elastic properties, the FFT computation methodology presented in the previous sections could be used to compute the effective bulk and shear moduli of 2-D or 3-D media. This extension is however left for future works to the benefit of the purely analytical results presented below.

First of all, we propose estimates of the effective moduli by direct extension of the heuristic method developed previously to derive the aforementioned analytic estimate (32) for the effective conductivity. Let us consider the PCW upper bound (relation (8)) for the effective bulk modulus. When cracks are located at inter-granular boundaries, the bulk modulus will

vanish when the crack density tends to its saturation value $\rho_{\text {sat }(d)}^{I G B}$. This limit value does not depend on the considered physical property but on the microstructure features only. Therefore, when cracks are located at inter-granular boundaries, a relevant estimate of the 
effective bulk modulus would be:

$$
\tilde{k}^{\mathrm{IGB}}(\rho)=k^{(1)}\left(\frac{1-\rho / \rho_{\operatorname{sat}(d)}^{I G B}}{1+\left(\beta_{k(d)}-1 / \rho_{\text {sat }(d)}^{I G B}\right) \rho}\right)
$$

Starting from the expression of the upper bound on the effective PCW shear modulus $\tilde{\mu}^{H S+}(\rho)$ (see Ponte-Castañeda \& Willis (1995)), a similar estimate can be derived for the effective shear modulus:

$$
\tilde{\mu}^{\mathrm{IGB}}(\rho)=\mu^{(1)}\left(\frac{1-\rho / \rho_{\text {sat }(d)}^{I G B}}{1+\left(\beta_{\mu(d)}-1 / \rho_{\text {sat }(d)}^{I G B}\right) \rho}\right)
$$

The crack density at saturation $\rho_{\text {sat }(d)}^{(I G B)}$ is given by relation (29). It depends on the size $R_{l}$ of the elementary cracks covering the inter-granular boundaries as well as the area per unit volume related to the inter-granular boundaries $-A_{V}^{(I G B)}$ - (or the length per unit surface related to the inter-granular boundaries in $\left.2-\mathrm{D}-L_{A}^{(I G B)}\right)$. In addition to this unique microstructural parameter $\rho_{\text {sat }(d)}$ (for a given dimension $d \in\{2,3\}$ ), the expressions of the coefficients $\left(\beta_{\mu(d)}, \beta_{k(d)}\right)$ are explicitly reported in Table 2 as a function of the elasticity coefficients of the solid. These coefficients and the microstructural parameter also depend on the shape of the elementary cracks covering inter-granular boundaries (respectively penny-shaped and ribbon-cracks for 3-D and 2-D cases).

\begin{tabular}{|l|c|c|}
\hline & $\beta_{\mu(d)}$ & $\beta_{k(d)}$ \\
\hline$d=2(2-D)$ & $\frac{\left(k^{(1)}+\mu^{(1)}\right)}{2 \mu^{(1)}} \pi$ \\
\hline$d=3(3-D)$ & $\frac{32\left(1-\nu^{(1)}\right)\left(5-\nu^{(1)}\right)}{45 \pi\left(2-\nu^{(1)}\right)}$ & $\frac{16}{9} \frac{\left(1-\left(\nu^{(1)}\right)^{2}\right)}{1-2 \nu^{(1)}}$ \\
\hline
\end{tabular}

Table 2: Expressions of the coefficients $\left(\beta_{\mu(d)}, \beta_{k(d)}\right)$ involved in the proposed estimations (34) and (35) of the effective moduli when cracks are located at inter-granular boundaries.

Alternatively, we can follow the so-called cross-property relations (see Torquato (2013)) to derive an upper bound of the effective bulk modulus from the estimates of the effective conductivity. For cracked media, these cross-property relations have been given by Gibiansky \& Torquato (1996a). For a given microstructure, the effective bulk modulus $\tilde{k}(\rho)$ is bounded by any estimate of the effective conductivity $\tilde{\sigma}(\rho)$ of the same microstructure:

$$
\frac{1}{\tilde{k}(\rho)}-\frac{1}{k^{(1)}} \geq \gamma_{d} \frac{\sigma^{(1)}}{k^{(1)}}\left(\frac{1}{\tilde{\sigma}(\rho)}-\frac{1}{\sigma^{(1)}}\right)
$$

where the coefficient $\gamma_{d}$ depends on the spatial dimension $d \in\{2,3\}$ and is given by:

$$
\gamma_{2}=\frac{\left(k^{(1)}+\mu^{(1)}\right)}{2 \mu^{(1)}} \quad \text { or } \quad \gamma_{3}=\frac{3 k^{(1)}}{2 \mu^{(1)}} \min \left\{1, \frac{1-\nu^{(1)}}{1+\nu^{(1)}}\right\}
$$

For the effective shear modulus, similar relations only exist for 2D-solids (see Gibiansky \& Torquato (1996b)). 
To go further, we first remark that relation (32) for the effective conductivity is equivalent to:

$$
\frac{1}{\tilde{\sigma}^{\mathrm{IGB}}(\rho)}-\frac{1}{\sigma^{(1)}}=\frac{1}{\sigma^{(1)}}\left(\frac{\rho \beta_{d}}{1-\rho / \rho_{\text {sat }(d)}^{I G B}}\right)
$$

As reported in Figures 9(b) and 10(b), the analytical estimate (32) for 3-D cases matches the simulated FFT results fairly well so that it can be considered as an exact result, at least for the studied microstructures. Therefore, injecting this analytic expression in the cross-property relation yields a bound. For 2-D cases, as the estimate (32) does not perfectly match FFT results, the tabulated numerical data related to FFT results should be used instead of relation (32) in the cross-property relation to derive a numerical upper bound of the 2-D effective bulk modulus.

Assuming that this last relation is an exact result for 3-D cases, we can inject it in equation (36) to derive the following expression of the new upper bound $\tilde{k}^{+}(\rho)$ (for $d=3$ ):

$$
\tilde{k}(\rho) \leq \tilde{k}^{+}(\rho)=k^{(1)}\left(\frac{1-\rho / \rho_{\text {sat }(3)}^{I G B}}{1+\left(\gamma_{3} \beta_{3}-1 / \rho_{\text {sat }(3)}^{I G B}\right) \rho}\right)
$$

Remarkably, this upper bound for the bulk modulus vanishes for $\rho=\rho_{\text {sat }(3)}^{I G B}$ coinciding with the aforementioned estimate in that limit. However, the slope at $\rho=0$ differs from the slope related to this estimate and is given by:

$$
\left(\frac{d \tilde{k}^{+}}{d \rho}\right)_{\rho=0}=-\gamma_{3} \beta_{3}
$$

This slope is higher than $\left(-\beta_{k(3)}\right)$ corresponding to the heuristic estimate as defined by relation (34). ${ }^{3}$ As a result, the estimate (34) lies below the upper bound $\tilde{k}^{+}(\rho)$ as given by relation (39).

To derive expressions of the effective moduli from the effective conductivity consistent with the initial slope, the cross-property relations proposed by Bristow (1960) can be used as an alternative. Starting from the "non-interacting" approximations of the effective properties, these cross-property relations have been obtained by substituting the crack density parameter by the effective conductivity. For instance for the bulk modulus (see Kachanov \& Sevostianov (2018) for a review), it yields:

$$
\frac{k^{(1)}-\tilde{k}^{\mathrm{NI}}(\rho)}{\tilde{k}^{\mathrm{NI}}(\rho)}=2 \frac{1-\left(\nu^{(1)}\right)^{2}}{1-2 \nu^{(1)}}\left(\frac{\sigma^{(1)}-\tilde{\sigma}^{\mathrm{NI}}(\rho)}{\tilde{\sigma}^{\mathrm{NI}}(\rho)}\right)
$$

${ }^{3}$ For a 3-D media, denoting by $\left|\nu^{(1)}\right|$ the absolute value of the Poisson ratio $\nu^{(1)}$ :

$$
\left(\frac{d \tilde{k}^{+}}{d \rho}\right)_{\rho=0}-\left(\frac{d \tilde{k}}{d \rho}\right)_{\rho=0}=\left(\frac{2\left(1-\left(\nu^{(1)}\right)^{2}\right)}{1-2 \nu^{(1)}}-\gamma_{3}\right) \beta_{3}=\frac{\left(1-\left|\nu^{(1)}\right|\right)\left(1+2 \nu^{(1)}\right)}{1-2\left|\nu^{(1)}\right|} \beta_{3}
$$

is always positive when $-1 \leq \nu^{(1)} \leq \frac{1}{2}$. 


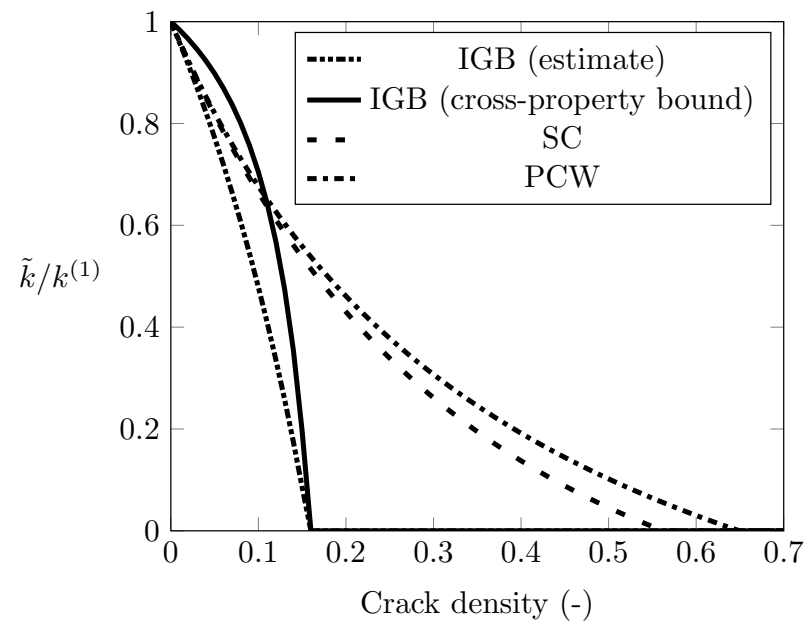

Figure 11: Normalized effective bulk modulus as a function of the crack density in 3-D cases with $\nu^{(1)}=0.3$ : comparison with analytical results given by the PCW upper bound ( $\mathrm{PCW}$, dot-dashed line), the self-consistent estimate (sc, loosely dashed line), the new "IGB" estimate defined by the relation (34) (IGB, densely dotdashed) and the bound defined by the cross-property relation (39) (solid line).

where the multiplicative coefficient equals the $\beta_{k(d)} / \beta_{d}$ ratio (relation (12) in section 2.2.1) which ensures that the initial slope of the bulk modulus is consistent with that of the dilute model when the slope of the effective conductivity satisfies this condition. In fact, starting from the more convenient expression (38) conjugated with the following expression of the effective bulk modulus,

$$
\frac{1}{\tilde{k}^{\mathrm{IGB}}(\rho)}-\frac{1}{k^{(1)}}=\frac{1}{k^{(1)}}\left(\frac{\rho \beta_{k(d)}}{1-\rho / \rho_{\text {sat }(d)}^{I G B}}\right)
$$

which is equivalent to (34), it is straightforward to demonstrate that the estimates (32) and (34) of the effective conductivity and bulk modulus (when cracks are located at inter-granular boundaries) satisfy also the cross-property relation (42) proposed by Bristow. This remark remains also true for the estimate of the effective shear modulus.

In Figure 11, these estimates and bounds of the effective bulk modulus of 3-D solids are plotted as a function of the crack density for a Poisson ratio of 0.3. The PCW upper bound and the self-consistent estimate (see section 2.2) are also reported in the same figure. As observed for the effective conductivity, the effective bulk modulus drops rapidly when

cracks are located at inter-granular boundaries. In addition, the estimate (34) yields results consistent with the bound (39) obtained from the cross-property relation (36).

\section{Conclusion}

A new methodology has been presented to model 2-D or 3-D cracked solids weakened at their inter-granular boundaries. The intersection of the inter-granular boundaries and localization 
disks in 2-D or spheres in 3-D leads to modeling randomly oriented cracks distributed along inter-granular boundaries. The coverage ratio of the inter-granular boundaries can be directly controlled by the density of the localization disks (or spheres).

Morphological effects have been highlighted in 2-D and in 3-D by studying the effect of the grains size to localization disks/spheres diameter ratio on the effective conductivity. For a given size of localization disks or spheres (regulating the size of elementary cracks) and a fixed inter-granular coverage ratio, increasing the grain size induces an increase in the effective conductivity by multiplying the number of thermal bridges between grains and therefore the possible paths for the heat flux.

In order to study the impact of the crack spatial distribution, the effective thermal conductivities of the 2-D and 3-D generated microstructures were compared against those of uniformly weakened solids. For uniform crack dispersions, the known PCW upper bound (applied to the conduction problem in this work) proves to be close to the full-field results while the differential scheme underestimates the effect of the cracks density on the effective conductivity when the crack density exceeds 0.5 . The properties of solids weakened at their inter-granular boundaries drop at much lower crack densities than those of solids with randomly distributed ribbon-cracks. The spatial distribution of cracks along inter-granular boundaries therefore induces a dramatic reduction in the effective thermal conductivity.

An analytical estimate of the effective conductivity of a solid weakened by micro-cracks located at inter-granular boundaries has also been proposed. It is based on an idea similar to that used by Sevostianov \& Kachanov (2019) which consists in restricting the possible location for inter-granular cracks in a conventional model but makes use of the PCW upper bound instead of the differential scheme. This new estimate agrees well with the simulated data for 2-D and 3-D media and it also improves on the model proposed by Sevostianov \& Kachanov (2019). This estimate can be directly used to compute effective properties such as electrical conduction and magnetic permeability. In addition, related theoretical results have also been provided to deliver estimates of the effective moduli of 2-D or 3-D elastic solids. These new estimates for the effective conductivity and elastic properties only depend on the size, the shape and the density of the elementary cracks, as well as the area per unit volume in 3-D (or the length per unit surface in 2-D) of the inter-granular network. In addition, these estimates yield results consistent with classical results when the crack density is low.

In a near future, these results will be used to model the effect of the cracks spatial distribution on the thermal properties of nuclear oxide fuels Meynard (2020). Still in the nuclear field, these results will also be used to model the improved thermal conductivity of enhanced accident tolerant fuels (such as micro-cell $\mathrm{UO}_{2}-$ Mo pellets Kim et al. (2015)) but the insulated cracks along inter-granular boundaries considered in this work will be replaced with highly conducting (metallic) materials.

From a theoretical viewpoint, the potential effect of some physical features of the intergranular network itself has to be more deeply studied in the future. Real microstructures often display a grain size distribution. In addition, the grain size may also be reduced or elongated in specific directions in connection with the fabrication process. Another aspect that influences the inter-granular network is the grain geometry. In real media the grains often have a more complex shape than polyhedral ones. Such a microstructure can be better 
approximated by a Johnson-Mehl tessellation, for instance, which leads to grains that are not necessarily convex and whose faces are not necessarily flat. Thereafter, the preliminary results related to the impact of the spatial distribution of inter-granular cracks on the effective mechanical properties would deserve to be consolidated by full-field computations and extended to properties related to nonlinear behaviors (plastic yield surface, ... ).

\section{Acknowledgements}

This work was partially developed within the framework of the MISTRAL joint research laboratory between the Aix-Marseille University, the French National Research Center (CNRS), Centrale Marseille and the French Alternative Energies and Atomic Energy Commission (CEA). The authors are grateful for the financial support provided by the CEA. The microstructures and the FFT computations were produced with version 1.4 of the VER software

and with version 1.8 of the TMFFT software (components of the PLEIADES platform), respectively. 


\section{Appendices}

\section{Appendix A Derivation of the upper estimate in Fig- ure 10 for $R_{l} / R \rightarrow 0$}

When cracks are located at inter-granular boundaries and when their size is very smallwith respect to the grain size (see an illustration for 2-D solids in Figure 5(a)), an upper estimate of the effective conductivity can be derived as detailed in this appendix.Its results are reported in Figure 10. The underlying idea is to replace a multi-cracked boundary by an equivalent homogeneous one. More precisely, the aim is to provide an effective thermal conductivity for a heat transfer across the interface, which describes the overall transfer through the numerous thermal bridges and isolating cracks, assuming there is a separation of length scale between these local heat flux fluctuations and the overall heat flux from a grain to its neighbor. Conductivity along directions parallel to the interface is not considered because, owing to the small thickness of the interfaces, heat flux along the essentially isolating interfaces is negligible with respect to the heat flux in the nearby solid. The required effective normal properties of the cracked interface are obtained by numerical periodic homogenization of the heterogeneous interface.

First we denote by $\tilde{\sigma}_{\text {sat }}\left(\sigma^{(2)} / \sigma^{(1)}\right)$ the effective conductivity of a solid where the conductivity of the inter-granular boundaries denoted by $\sigma^{(2)}$ differs from the one of the solid $\sigma^{(1)}$. For the microstructures considered in this paper and when $\sigma^{(2)} \leq \sigma^{(1)}$, the tabulated function $\tilde{\sigma}_{\text {sat }}\left(\sigma^{(2)} / \sigma^{(1)}\right)$ is reported in Figure 8 .

When the crack size is much smaller than the grain size (i.e $R_{l} / R \ll 1$ ), this function can be used to evaluate the effective conductivity of a granular material cracked at its intergranular boundaries by setting $\sigma^{(2)}$ to be the local effective conductivity of the inter-granular boundaries weakened by micro-cracks. Obviously, this effective conductivity depends on the inter-granular coverage ratio $c_{\text {cov }}^{(2)}$ and ranges from $\sigma^{(1)}\left(c_{c o v}^{(2)}=0\right)$ to zero $\left(c_{c o v}^{(2)}=1\right)$. To estimate $\sigma^{(2)}$ between these two extreme values, we consider a periodic unit cell. For 2-D cases, overlapping cracks are randomly located on a horizontal inter-granular boundary as shown in Figure 12(a). The crack thickness $e$ is small compared with the size of the unit cell $L_{u c}$ while the inter-granular coverage ratio is given by the ratio between the total length covered by the cracks and the size of the unit cell. Similar microstructures are generated in 3 -D cases by considering a periodic dispersion of penny-shaped cracks on horizontal planes (see Figure 12(b)). In this way, we can account for the inter-granular coverage ratio and the morphology of the dispersion of small cracks over the interface in the numerical determination of the effective conductivity in the direction normal to the cracks, corresponding to the vertical direction in Figure 12(a), and the normal direction to the cracked interface plane shown in Figure 12(b).

The simulated effective normal conductivities in 2-D and 3-D cases denoted $\tilde{\sigma}_{u c(d)}^{\perp}\left(c_{c o v}^{(2)}\right)$ are reported in Figure A.13. The size of the square or cubic unit cell is chosen large with respect to the cracks size, approximately 150 and 40 times larger for 2-D and 3-D cases respectively.

As expected, the effective conductivity of the unit cell decreases when the inter-granular coverage ratio increases. This decrease is slightly less marked for 2-D solids, probably due 


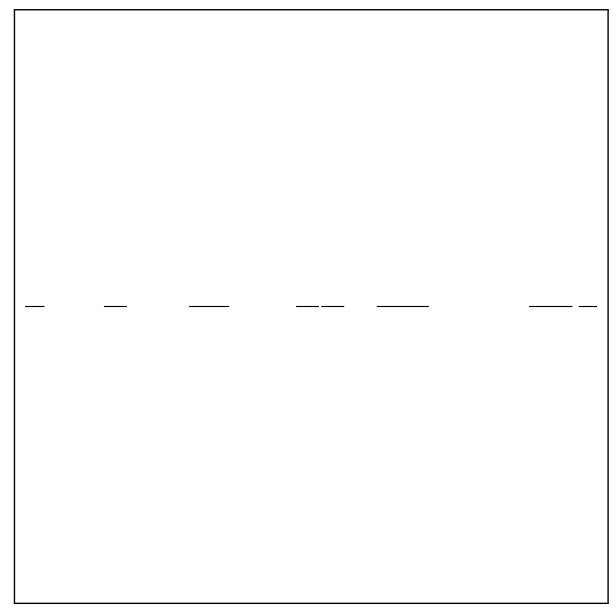

(a)

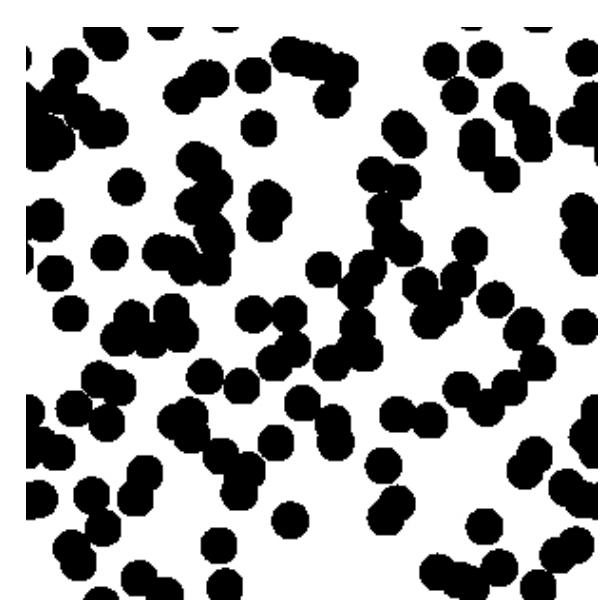

(b)

Figure A.12: The periodic unit cells used to compute $\tilde{\sigma}_{u c(2)}^{\perp}$ in 2-D cases (Figure (a)) and 3-D cases (plane of the aligned cracks, Figure (b)). $c_{\text {cov }}^{(2)} \approx 0.4$ for both microstructures.

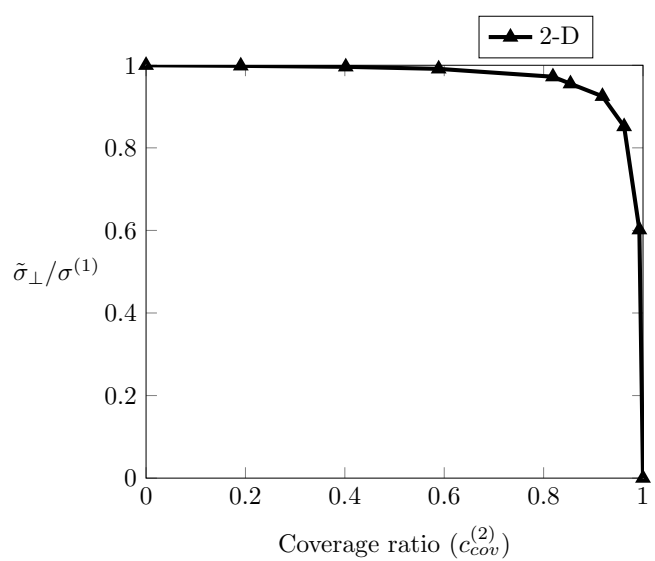

(a)

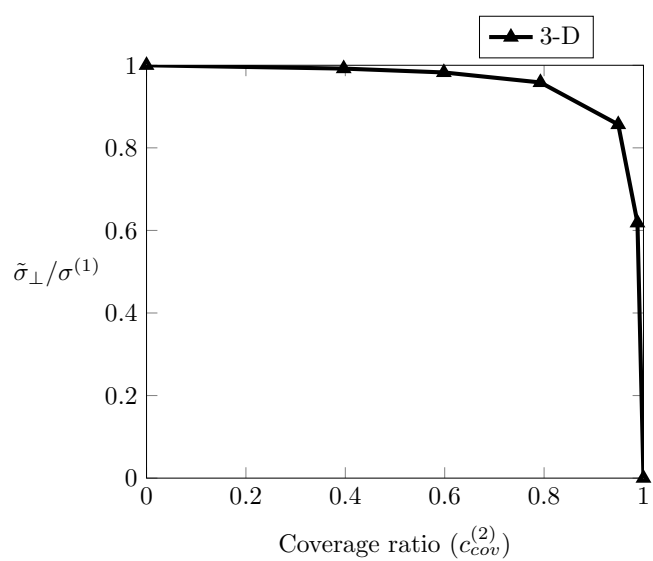

(b)

Figure A.13: The effective normal conductivity along a direction perpendicular to the cracks as a function of the inter-granular coverage ratio for 2-D solids (Figure (a)) and 3-D solids (Figure (b)).

to the lower ratio between the length of the unit cell and the crack size adopted for 3-D computations.

The obtained conductivity is relative to a periodic body of finite size. We still need to convert it into the effective conductivity $\sigma^{(2)}$ of the inter-granular boundary with thickness $e$. To do so, we consider the same unit cell but substitute the horizontal surface containing the aligned cracks with a homogeneous material of conductivity $\sigma^{(2)}$, defined in such a way that both unit cells exhibit the same normal effective conductivity. The solution of this last problem is straightforward as the heat flux is actually uniform so that the effective thermal conductivity coincides with the lower Wiener bound and reads:

$$
\frac{1}{\tilde{\sigma}_{u c(d)}^{\perp}\left(c_{c o v}^{(2)}\right)}=\frac{1-c^{(I G B)}}{\sigma^{(1)}}+\frac{c^{(I G B)}}{\sigma^{(2)}}
$$


where $c^{(I G B)}$ is the volume fraction of the interface in the unit cell, computed as the ratio between the crack thickness $e$ and the length of the unit-cell.

This relation can be easily inverted to obtain the effective conductivity $\sigma^{(2)}$ of the interface as a function of the inter-granular coverage ratio $c_{\text {cov }}^{(2)}$ :

$$
\sigma^{(2)}\left(c^{(I G B)}, c_{c o v}^{(2)}, \sigma^{(1)}\right)=\frac{c^{(I G B)} \sigma^{(1)} \tilde{\sigma}_{u c(d)}^{\perp}\left(c_{c o v}^{(2)}\right)}{\sigma^{(1)}-\left(1-c^{(I G B)}\right) \tilde{\sigma}_{u c(d)}^{\perp}\left(c_{\text {cov }}^{(2)}\right)}
$$

Finally, for a given inter-granular coverage ratio $c_{c o v}^{(2)}$, once the conductivity $\sigma^{(2)}$ of the IGB is estimated by relation (A.2), the upper estimate is obtained from the tabulated function $\tilde{\sigma}_{\text {sat }}\left(\sigma^{(2)} / \sigma^{(1)}\right)$ (random dispersion of cracks along inter-granular boundaries).

Note that the thickness of the interface in the unit cell could have been different from that used in the FFT computations of the granular microstructure. The quantity actually under interest is the normal thermal conductance of the interface $c_{\text {cov }}^{(2)} / e$. If another interface thickness $e^{\prime}$ would be used, the conductivity would need to be adjusted such that $c_{\text {cov }}^{(2)^{\prime}} / e^{\prime}=$ $c_{\text {cov }}^{(2)} / e$. Relation (A.1) would still hold as long as $e$ and $e^{\prime}$ are small with respect to the unit-cell length $L_{u c}$. Note also that this approach could be enriched by the evaluation of the in-plane conductivity of the homogenized interface, which would then be attributed anisotropic properties. This would however require FFT computations much more complex to implement, as local anisotropic properties of the interface would then need to be defined in connection with its local orientation, for a presumably limited improvement.

\section{References}

Anderson, D. G. 1965. Iterative procedures for nonlinear integral equations. Journal of the ACM (JACM), 12(4), 547-560.

Bilger, N., Auslender, F., Bornert, M., Michel, J. C., Moulinec, H., Suquet, P., \& Zaoui, A. 2005. Effect of a nonuniform distribution of voids on the plastic response of voided materials: a computational and statistical analysis. International Journal of Solids and Structures, 42(2), 517-538.

Bristow, J. R. 1960. Microcracks, and the static and dynamic elastic constants of annealed and heavily cold-worked metals. British Journal of Applied Physics, 11(2), 81.

Budiansky, B., \& O'Connell, R. J. 1976. Elastic moduli of a cracked solid. International journal of Solids and structures, 12(2), 81-97.

Duan, H. L., Karihaloo, B. L., Wang, J., \& Yi, X. 2006. Effective conductivities of heterogeneous media containing multiple inclusions with various spatial distributions. Physical Review B, 73(17), 174203.

Gasnier, J. B., Willot, F., Trumel, H., Jeulin, D., \& Besson, J. 2018. Thermoelastic properties of microcracked polycrystals. Part I: Adequacy of Fourier-based methods for cracked elastic bodies. International Journal of Solids and Structures, 155, 248-256. 
Gibiansky, L. V., \& Torquato, S. 1996a. Bounds on the effective moduli of cracked materials. Journal of the Mechanics and Physics of Solids, 44(2), 233-242.

Gibiansky, L. V., \& Torquato, S. 1996b. Connection between the conductivity and bulk modulus of isotropic composite materials. Proceedings of the Royal Society of London. Series A: Mathematical, Physical and Engineering Sciences, 452(1945), 253-283.

Grechka, V., Vasconcelos, I., \& Kachanov, M. 2006. The influence of crack shape on the effective elasticity of fractured rocks. Geophysics, 71(5), D153-D160.

Hashin, Z. 1988. The differential scheme and its application to cracked materials. Journal of the Mechanics and Physics of Solids, 36(6), 719-734.

Hashin, Z., \& Shtrikman, S. 1962. A variational approach to the theory of the effective magnetic permeability of multiphase materials. Journal of applied Physics, 33(10), 31253131.

Hoenig, A. 1983. Thermal conductivities of a cracked solid. Journal of Composite Materials, 17(3), 231-237.

Johnson, WA, \& Mehl, RF. 1939. Reaction kinetics in processes of nucleation and growth: AIME Tech.

Kachanov, M. 1992. Effective elastic properties of cracked solids: critical review of some basic concepts. Applied Mechanics Reviews, 45(8), 304-335.

Kachanov, Mark, \& Sevostianov, Igor. 2018. Micromechanics of materials, with applications. Vol. 249. Springer.

Kim, D. J., Rhee, Y. W., Kim, J. H., Kim, K. S., Oh, J. S., Yang, J. H., Koo, Y. H., \& Song, K. W. 2015. Fabrication of micro-cell $\mathrm{UO}_{2}$-Mo pellet with enhanced thermal conductivity. Journal of Nuclear Materials, 462, 289-295.

Kim, I. C., \& Torquato, S. 1992. Effective conductivity of suspensions of overlapping spheres. Journal of applied physics, 71(6), 2727-2735.

Lantuéjoul, C. 2002. Geostatistical Simulation: Models and Algorithms. Springer.

Li, J., Tian, X., \& Abdelmoula, R. 2012a. A damage model for crack prediction in brittle and quasi-brittle materials solved by the FFT method. International journal of fracture, $\mathbf{1 7 3}(2), 13-146$.

Li, J., Meng, S., Tian, X., Song, F., \& Jiang, C. 2012b. A non-local fracture model for composite laminates and numerical simulations by using the FFT method. Composites Part B: Engineering, 43(3), $961-971$.

Maxwell, J. C. 1873. A treatise on electricity and magnetism. Vol. 1. Clarendon press. 
Meynard, J. 2020. Influence de la taille, de la morphologie et de la distribution spatiale des pores sur la conductivité thermique de céramiques UO2. Ph.D. thesis, Aix-Marseille Université.

Mori, T., \& Tanaka, K. 1973. Average stress in matrix and average elastic energy of materials with misfitting inclusions. Acta metallurgica, 21(5), 571-574.

Moulinec, H., \& Suquet, P. 1998. A numerical method for computing the overall response of nonlinear composites with complex microstructure. Computer methods in applied mechanics and engineering, 157(1-2), 69-94.

Ponte-Castañeda, P., \& Willis, J. R. 1995. The effect of spatial distribution on the effective behavior of composite materials and cracked media. Journal of the Mechanics and Physics of Solids, 43(12), 1919-1951.

Pouya, A., \& Ghoreychi, M. 2001. Determination of rock mass strength properties by homogenization. International Journal for Numerical and Analytical Methods in Geomechanics, 25(13), 1285-1303.

Ramière, I., \& Helfer, T. 2015. Iterative residual-based vector methods to accelerate fixed point iterations. Computers \& Mathematics with Applications, 70(9), 2210-2226.

Sevostianov, I., \& Kachanov, M. 2019. On the effective properties of polycrystals with intergranular cracks. International Journal of Solids and Structures, 156, 243-250.

Sevostianov, I., Agnihotri, G., \& Garay, J. F. 2004. On connections between 3-D microstructures and their 2-D images. International journal of fracture, 126(3), 65-72.

Shafiro, B., \& Kachanov, M. 2000. Anisotropic effective conductivity of materials with nonrandomly oriented inclusions of diverse ellipsoidal shapes. Journal of applied physics, 87(12), 8561-8569.

Torquato, S. 2013. Random heterogeneous materials: microstructure and macroscopic properties. Vol. 16. Springer Science and Business media.

Torquato, S., Uche, O. U., \& Stillinger, F. H. 2006. Random sequential addition of hard spheres in high Euclidean dimensions. Physical Review E, 74(6).

Toth, A., Kelley, C. T., Slattery, S., Hamilton, S., Clarno, K., \& Pawlowski, R. 2015. Analysis of Anderson acceleration on a simplified neutronics/thermal hydraulics system. Page 34 of: Joint International Conference on Mathematics and Computation (M\&C), Supercomputing in Nuclear Applications (SNA), and the Monte Carlo (MC) Method.

Wiener, O. 1912. Die Theorie des Mischkorpers fur das Feld der stationaren Stromung. Abhandlungen der Sachsischen Gesellschaft der Akademischen Wissenschaften in Mathematik und Physik, 32, 507-604. 
Willot, F., \& Pellegrini, Y-P. 2008. Fast Fourier transform computations and build-up of plastic deformation in 2D,elastic-perfectly plastic, pixelwise-disordered porous media. Page 443-449 of: Jeulin, D., \& Forest, S. (eds), Continuum Models and Discrete Systems CMDS 11. École des Mines, Paris. 